\title{
MODULATING FUNCTIONS BASED ALGORITHM FOR THE ESTIMATION OF THE COEFFICIENTS AND DIFFERENTIATION ORDER FOR A SPACE-FRACTIONAL ADVECTION-DISPERSION EQUATION*
}

\author{
ABEER ALDOGHAITHER ${ }^{\dagger}$, DA-YAN LIU $^{\ddagger}$, AND TAOUS-MERIEM LALEG-KIRATI $^{\S}$
}

\begin{abstract}
In this paper, a new method, based on the so-called modulating functions, is proposed to estimate average velocity, dispersion coefficient, and differentiation order in a space-fractional advection-dispersion equation, where the average velocity and the dispersion coefficient are spacevarying. First, the average velocity and the dispersion coefficient are estimated by applying the modulating functions method, where the problem is transformed into a linear system of algebraic equations. Then, the modulating functions method combined with a Newton's iteration algorithm is applied to estimate the coefficients and the differentiation order simultaneously. The local convergence of the proposed method is proved. Numerical results are presented with noisy measurements to show the effectiveness and robustness of the proposed method. It is worth mentioning that this method can be extended to general fractional partial differential equations.
\end{abstract}

Key words. fractional advection-dispersion equation, parameter identification, fractional order estimation, modulating functions method

AMS subject classifications. 35R11, 35R30

DOI. $10.1137 / 15 \mathrm{M} 1008993$

1. Introduction. Fractional calculus has been introduced as an efficient tool for modeling many physical phenomena thanks to its memory and hereditary properties [7, 23]. For instance, fractional models have been successfully used to describe anomalous diffusion processes such as contaminants transport in soil, oil flow in porous media, and groundwater flow $[28,24,29]$. These models capture important features of particles transport such as particles with velocity variation and long-rest periods [23].

The direct problem for fractional differential equations has been widely studied (see $[16,8,18,3,26])$, while work on inverse problems has been less considered. In this paper, we consider an inverse problem for the following space-fractional advectiondispersion equation with initial and Dirichlet boundary conditions describing, for example, solute transport in porous media [30]: for any $0 \leq x \leq L$ and $t>0$,

$$
\frac{\partial c(x, t)}{\partial t}=-\nu(x) \frac{\partial c(x, t)}{\partial x}+d(x) \frac{\partial^{\alpha} c(x, t)}{\partial x^{\alpha}}+r(x, t),
$$

*Submitted to the journal's Methods and Algorithms for Scientific Computing section February 17, 2015; accepted for publication (in revised form) August 27, 2015; published electronically December 1, 2015. This research was supported by the King Abdullah University of Science and Technology (KAUST).

http://www.siam.org/journals/sisc/37-6/M100899.html

$\dagger$ Computer, Electrical, and Mathematical Sciences and Engineering Division, King Abdullah University of Science and Technology (KAUST), Thuwal, 53955-6900, Kingdom of Saudi Arabia (abeer.aldoghaither@kaust.edu.sa).

${ }^{\ddagger}$ INSA Centre Val de Loire, Université d’Orléans, PRISME EA 4229, Bourges, France (dayan.liu@ insa-cvl.fr).

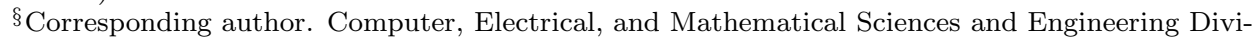
sion, King Abdullah University of Science and Technology (KAUST), Thuwal, 53955-6900, Kingdom of Saudi Arabia (taousmeriem.laleg@kaust.edu.sa).

A2813 


$$
\left\{\begin{array}{l}
c(x, 0)=g_{0}(x), \\
c(0, t)=0, \\
c(L, t)=0,
\end{array}\right.
$$

where $c$ is the solute concentration, $\nu$ and $d$ are the average velocity and the dispersion coefficient, respectively, which we suppose positive, fixing the direction of flow, $r$ is the source term, and $\alpha$ is the differentiation order for the space derivative with $1<\alpha \leq 2$. The fractional derivative $\frac{\partial^{\alpha}}{\partial x^{\alpha}}$ is a Riemann-Liouville derivative of order $\alpha$ which will be defined later in Definition 2.1. The coefficients $\nu(x), d(x)$ and the source term $r(x, t)$ are such that (1.1) has a unique and sufficiently smooth solution under the initial and boundary conditions (1.2).

We are interested in identifying average velocity, dispersion coefficient, and differentiation order for the space-fractional advection-dispersion equation defined by (1.1)-(1.2). We suppose that the concentration $c$ and the flux $\frac{\partial c}{\partial t}$ are unknown except at a final time $t=T$, where they can be measured:

$$
g_{1}(x)=c(x, T)+\xi_{1}, \quad g_{2}(x)=\frac{\partial c(x, T)}{\partial t}+\xi_{2},
$$

where $\xi_{1}$ and $\xi_{2}$ are noise contaminating the data.

This inverse problem falls in the category of parameter identification, where we use measurable data to infer properties related to the structure of some physical systems. Since the space-fractional advection-dispersion equation is usually used to model solute transport in heterogeneous porous media [24], identifying parameters for such an equation is important to understand how chemical or biological contaminates are transported throughout a surface aquifer system. For instance, an estimate of the differentiation order in a ground water contaminant transport model can provide information about soil properties, such as the heterogeneity of the media [23].

Estimating coefficients for fractional differential equations is not a trivial problem especially if the coefficients are not constant. Moreover, the problem becomes more challenging when it involves the identification of the differentiation order, where usually standard approaches fail [21]. Some progress has been made for the estimation of the coefficient for the fractional diffusion equation $[10,1,2,17]$, while the coefficient estimation problem for the space-fractional advection-dispersion equation has not been paid much attention. For instance, Zhang et al. [30] considered an inverse problem based on optimization for simultaneous identification of multiple parameters in a space-fractional advection-dispersion equation. This inverse problem has been solved numerically using the optimal perturbation regularization algorithm introduced by $\mathrm{Chi}, \mathrm{Li}$, and Jia [4]. However, the sensitivity of the algorithm depends on the regularization parameter, the numerical differential step, and the initial iteration. In their study, they considered Tikhonov regularization to treat the ill posedness of the problem. In spite of the importance, to the best of our knowledge, except for [30] there is no published work on the parameter identification problem for the space-fractional advection-dispersion equation especially in the case where the differentiation order is unknown.

In our study, we propose a novel approach, where the modulating functions method (MFM) is combined with a Newton's iteration algorithm to estimate all three parameters simultaneously. The MFM was first introduced in 1954 by Shinbrot [25] to identify parameters for linear dynamical systems, then it was used in many applications such as signal processing and control theory (see, e.g., [5, 27, 22, 6, 12, 14]). For 
instance, Fedele and Coluccio [6] presented a recursive frequency estimation scheme based on trigonometric and spline-type modulating functions. Janiczek [9] generalized the MFM to the fractional differential equations, where he aimed to reduce the fractional order to an integer order in a noise free case. Liu et al. [14] applied the MFM to identify unknown coefficients for a class of fractional order linear systems. Furthermore, Sadabadi, Shafiee, and Karrari [22] estimated the parameters for a two dimensional continuous-time system, based on a two dimensional modulating functions approach. Recently, the MFM has been extended to the numerical differentiation problem by introducing the so-called generalized modulating functions $[15,13]$. However, to the best of our knowledge, there is no study generalizing the MFM to fractional partial differential equations. Moreover, this method has never been used to estimate the differentiation order.

Standard approaches to solving parameter identification problems are based on optimization. However, in this paper we introduce a new method where the MFM is combined with an iterative Newton's algorithm to estimate simultaneously the coefficients and the differentiation order for a space-fractional advection-dispersion equation. The use of the modulating functions has several advantages. First, the problem of estimating the average velocity and the dispersion coefficient is simplified to solving a system of algebraic equations, where the estimations of the unknown parameters can be given in closed form by an integral formula. Second, initial values, which are usually unknown in most real life applications, are not required in this approach. Third, instead of solving the direct problem and computing the fractional derivative of the solution of the partial differential equation, the fractional derivatives of the modulating functions are computed. The latter can be simpler and calculated analytically if the modulating functions are well-chosen. Moreover, the proposed two stage approach which combines the MFM with an iterative Newton's algorithm to estimate the coefficient and the differentiation order simultaneously provides an efficient and robust method to compute the gradient needed for the Newton's method. Furthermore, the proposed method is independent of the nature of the partial differential equation, which, therefore, can easily be extended to identify the coefficients and the differentiation order of other fractional partial differential equations.

This paper is organized as follows: in section 2, the definition and some properties of the fractional derivatives and the modulating functions will be introduced. In section 3, the MFM is applied to the space-fractional advection-dispersion equation, where the average velocity and the dispersion coefficient are estimated by solving a system of algebraic equations. Then in section 4, the MFM is combined with the first order Newton's method to estimate all three parameters simultaneously. In section 5 , the local convergence of the proposed two stage algorithm is proved. In section 6 , numerical results are presented to illustrate the efficiency and robustness of the method. The obtained results are discussed in section 8. Finally, concluding remarks are provided towards the end.

2. Preliminaries. In this section, we recall the definition of the RiemannLiouville fractional derivative, the modulating functions, and some of their useful properties.

DEFINITION 2.1 (see [19, p. 62]). The $\alpha$ th order Riemann-Liouville fractional derivative of a function $f \in C^{n}(\mathbb{R})$, with $\alpha \in \mathbb{R}_{+}$, is defined as follows: for all $x \in \mathbb{R}$, (2.1)

$$
\frac{\partial^{\alpha}}{\partial x^{\alpha}} f(x)=\frac{1}{\Gamma(n-\alpha)} \frac{d^{n}}{d x^{n}} \int_{0}^{x}(x-\tau)^{n-\alpha-1} f(\tau) \mathrm{d} \tau, \quad n-1 \leq \alpha<n, \quad n \in \mathbb{N}^{*},
$$


where $\Gamma$ is the classical gamma function.

Definition 2.2 (see [20]). A function $\phi \in C^{k}([a, b])$, defined over the interval $[a, b]$, is called a modulating function of order $k$ with $k \in \mathbb{N}^{*}$ if

$$
\phi^{(i)}(a)=\phi^{(i)}(b)=0, \quad i=0,1, \ldots, k-1 .
$$

The following lemma describes a useful property of the modulating function. This lemma was obtained by applying the convolution theorem of the Laplace transform. A detailed proof can be found in [14].

Lemma 2.3 (see [14]). If the ath order Riemann-Liouville derivative of $f \in$ $C^{n}(\mathbb{R})$ exists where $n-1 \leq \alpha<n$, and $g$ is an $n$th order modulating function defined on $[0, I]$, then we have

$$
\int_{0}^{I} g(I-x) \frac{\partial^{\alpha} f(x)}{\partial x^{\alpha}} \mathrm{d} x=\int_{0}^{I} \frac{\partial^{\alpha} g(x)}{\partial x^{\alpha}} f(I-x) \mathrm{d} x .
$$

Next, we introduce the derivative with respect to the fractional order for the Riemann-Liouville derivative.

Proposition 2.4. If the $\alpha$ th order Riemann-Liouville derivative of $f$ exists where $n-1 \leq \alpha<n$, then the derivative of $\frac{\partial^{\alpha} f}{\partial x^{\alpha}}$ with respect to $\alpha$ is given by

$$
\frac{\partial}{\partial \alpha} \frac{\partial^{\alpha} f(x)}{\partial x^{\alpha}}=\psi_{0}(n-\alpha) \frac{\partial^{\alpha} f(x)}{\partial x^{\alpha}}-\frac{1}{\Gamma(n-\alpha)} \frac{d^{n}}{d x^{n}} \int_{0}^{x}(x-\tau)^{n-\alpha-1} \ln (x-\tau) f(\tau) \mathrm{d} \tau,
$$

where $\psi_{0}(n-\alpha)=\frac{\Gamma^{\prime}(n-\alpha)}{\Gamma(n-\alpha)}$.

In this paper, we suppose that the following assumptions on the modulating function $\phi$ hold:

H1. The function $\phi(x) \in C^{2}([0, L])$.

H2. The function $\frac{\partial}{\partial \alpha} \frac{\partial^{\alpha} \phi}{\partial x^{\alpha}}:[0, L] \rightarrow \mathbb{R}$ is continuous.

3. Modulating functions method for estimating the average velocity and the dispersion coefficient. In this section, the MFM is applied to estimate the average velocity and the dispersion coefficient by assuming that the differentiation order is known.

Let $\left\{f_{k}\right\}_{k=1}^{\infty}$ and $\left\{p_{k}\right\}_{k=1}^{\infty}$ be sets of basis functions where the average velocity $\nu$ and the dispersion coefficient $d$ can be written as $\nu(x)=\sum_{k=1}^{\infty} \nu_{k} f_{k}(x)$ and $d(x)=$ $\sum_{k=1}^{\infty} d_{k} p_{k}(x)$, where $\nu_{k}$ and $d_{k}$ are the basis coefficients.

Proposition 3.1. For $0<L_{1} \leq L$, let $\left\{\phi_{m}\right\}_{m=1}^{M}$ be a set of $M$ modulating functions of at least order 2 defined on the interval $\left[0, L_{1}\right]$ where $M \geq K_{1}+K_{2}$. Then, the unknown coefficients $\nu$ and $d$ can be estimated by

$$
\tilde{\nu}(x)=\sum_{k=1}^{K_{1}} \tilde{\nu}_{k} f_{k}(x), \quad \tilde{d}(x)=\sum_{k=1}^{K_{2}} \tilde{d}_{k} p_{k}(x),
$$

where the parameters $\left\{\tilde{\nu}_{k}\right\}_{k=1}^{K_{1}}$ and $\left\{\tilde{d}_{k}\right\}_{k=1}^{K_{2}}$ are given by the solution to the following linear system:

$$
\mathbf{Q X}=\mathbf{Y}
$$

Copyright (C) by SIAM. Unauthorized reproduction of this article is prohibited. 
with

$(3.3)$

$$
\mathbf{Q}=\left[\begin{array}{cccc}
q_{11} & q_{12} & \cdots & q_{1 K_{1}+K_{2}} \\
q_{21} & q_{22} & \cdots & q_{2 K_{1}+K_{2}} \\
\vdots & \vdots & \ddots & \vdots \\
q_{M 1} & q_{M 2} & \cdots & q_{M K_{1}+K_{2}}
\end{array}\right], \quad \mathbf{X}=\left[\begin{array}{c}
\tilde{\nu}_{1} \\
\tilde{\nu}_{2} \\
\vdots \\
\tilde{\nu}_{K_{1}} \\
\tilde{d}_{1} \\
\tilde{d}_{2} \\
\vdots \\
\tilde{d}_{K_{2}}
\end{array}\right], \quad \text { and } \quad \mathbf{Y}=\left[\begin{array}{c}
y_{1} \\
y_{2} \\
\vdots \\
y_{M}
\end{array}\right]
$$

where

$$
q_{m k}= \begin{cases}\int_{0}^{L_{1}} \frac{\partial\left[f_{k}(x) \phi_{m}\left(L_{1}-x\right)\right]}{\partial x} c(x, t) \mathrm{d} x, & k=1,2, \ldots, K_{1}, \\ \int_{0}^{L_{1}} \frac{\partial^{\alpha} \hat{\phi}_{m k}(x)}{\partial x^{\alpha}} c\left(L_{1}-x, t\right) \mathrm{d} x, & k=K_{1}+1, K_{1}+2, \ldots, K_{1}+K_{2},\end{cases}
$$

$$
y_{m}=-\int_{0}^{L_{1}} \phi_{m}\left(L_{1}-x\right)\left[r(x, t)-\frac{\partial c(x, t)}{\partial t}\right] \mathrm{d} x
$$

$\hat{\phi}_{m k}\left(L_{1}-x\right)=p_{k}(x) \phi_{m}\left(L_{1}-x\right), m=1, \ldots, M$.

Proof. Let $\left\{\phi_{m}(x)\right\}_{m=1}^{M}$ be a set of $M$ modulating functions of at least order 2 and consider (1.1). Then, by multiplying the modulating functions $\phi_{m}\left(L_{1}-\cdot\right)$ for $m=1, \ldots, M$ to $(1.1)$ and by integrating over the interval $\left[0, L_{1}\right]$, we get

$$
\begin{aligned}
\int_{0}^{L_{1}} \phi_{m}( & \left.L_{1}-x\right) \frac{\partial c(x, t)}{\partial t} \mathrm{~d} x=-\int_{0}^{L_{1}} \nu(x) \phi_{m}\left(L_{1}-x\right) \frac{\partial c(x, t)}{\partial x} \mathrm{~d} x \\
& +\int_{0}^{L_{1}} d(x) \phi_{m}\left(L_{1}-x\right) \frac{\partial^{\alpha} c(x, t)}{\partial x^{\alpha}} \mathrm{d} x+\int_{0}^{L_{1}} \phi_{m}\left(L_{1}-x\right) r(x, t) \mathrm{d} x
\end{aligned}
$$

Substituting (3.1) into (3.6), gives

$$
\begin{aligned}
-\int_{0}^{L_{1}} \phi_{m} & \left(L_{1}-x\right)\left[r(x, t)-\frac{\partial c(x, t)}{\partial t}\right] \mathrm{d} x \\
= & -\sum_{k=1}^{K_{1}} \tilde{\nu}_{k} \int_{0}^{L_{1}} f_{k}(x) \phi_{m}\left(L_{1}-x\right) \frac{\partial c(x, t)}{\partial x} \mathrm{~d} x \\
& +\sum_{k=1}^{K_{2}} \tilde{d}_{k} \int_{0}^{L_{1}} \hat{\phi}_{m k}\left(L_{1}-x\right) \frac{\partial^{\alpha} c(x, t)}{\partial x^{\alpha}} \mathrm{d} x
\end{aligned}
$$

where $\hat{\phi}_{m k}(L-x)=p_{k}(x) \phi_{m}\left(L_{1}-x\right)$. 
Applying integration by parts and Lemma 2.3 yields

$$
\begin{aligned}
-\int_{0}^{L_{1}} \phi_{m}\left(L_{1}-x\right)\left[r(x, t)-\frac{\partial c(x, t)}{\partial t}\right] \mathrm{d} x \\
=+\sum_{k=1}^{K_{1}} \tilde{\nu}_{k} \int_{0}^{L_{1}} \frac{\partial\left[f_{k}(x) \phi_{m}\left(L_{1}-x\right)\right]}{\partial x} c(x, t) \mathrm{d} x \\
+\sum_{k=1}^{K_{2}} \tilde{d}_{k} \int_{0}^{L_{1}} \frac{\partial^{\alpha} \hat{\phi}_{m k}(x)}{\partial x^{\alpha}} c\left(L_{1}-x, t\right) \mathrm{d} x,
\end{aligned}
$$

where the boundary conditions are eliminated by the properties of the used modulating functions. Finally, the unknown parameters $\left\{\tilde{\nu}_{k}\right\}_{k=1}^{K_{1}}$ and $\left\{\tilde{d}_{k}\right\}_{k=1}^{K_{2}}$ can be estimated by solving the linear system given in (3.2).

Remark 3.1. In the previous proposition if $M=K_{1}+K_{2}$, which means that the number of modulating functions and the number of unknown parameters are equal, then if $\mathbf{Q}$ is nonsingular, the system (3.2) has a unique solution given by $\mathbf{X}=\mathbf{Q}^{-1} \mathbf{Y}$. On the other hand, if $M>K_{1}+K_{2}$, then system (3.2) is overdetermined and the solution can be obtained using the least square method as $\mathbf{X}=\left(\mathbf{Q}^{\mathbf{T}} \mathbf{Q}\right)^{-1} \mathbf{Q}^{\mathbf{T}} \mathbf{Y}$. Moreover, if the number of terms in the expansion is large, regularization may be needed to solve the system. This may be the case, for example, when both parameters $\nu$ and $d$ are nonpolynomial, in which case the number of terms in the polynomial basis expansion is large.

The next result illustrates the particular case where $\nu$ and $d$ are constants.

Corollary 3.2. For $0<L_{1} \leq L$, let $\left\{\phi_{m}(x)\right\}_{m=1}^{M}$ be a set of at least second order modulating functions defined on the interval $\left[0, L_{1}\right]$ where $2 \leq M$, then the solution of the following linear system gives the estimations of the parameters $\nu$ and $d$ :

$$
\mathbf{P}\left[\begin{array}{l}
\nu \\
d
\end{array}\right]=\mathbf{Y},
$$

where

with

$$
\mathbf{P}=[\mathbf{A} \mid \mathbf{B}] \quad \text { and } \quad \mathbf{Y}=\left[\begin{array}{c}
y_{1} \\
y_{2} \\
\vdots \\
y_{M}
\end{array}\right]
$$

for $m=1,2, \ldots, M$.

Proof. The proof can be obtained as in Proposition 3.1, where the coefficients $\nu(x)$ and $d(x)$ are replaced by the constants coefficients $\nu$ and $d$. 
4. Parameter and differentiation order estimation. In this section, the MFM is combined with a Newton's-type method to simultaneously estimate $\nu, d$, and $\alpha$. Without loss of generality, from now on we will assume that the average velocity and the dispersion coefficient are constant. However, the proposed algorithm can be applied similarly for spatial-varying coefficients.

4.1. Combined Newton's and modulating functions method to estimate $\boldsymbol{d}, \boldsymbol{\nu}$, and $\boldsymbol{\alpha}$. Order of differentiation is usually unknown and often challenging to estimate. However, by using the concept of modulating functions, this difficulty is greatly reduced. Due to the nature of the problem it is demanding that we split the solution algorithm into two stages: the first stage solves the previous problem described in section 3, and the second stage deals with solving a nonlinear equation. Applying the modulating functions greatly simplifies the second stage of the algorithm, where we avoid solving the direct problem at each iteration and the nonlinear equation problem will depend only on one variable $\alpha$. It also allows us to compute the gradient in an efficient way.

Now, we introduce the two stage algorithm to estimate $\nu, d$, and $\alpha$.

Stage 1. In this stage, we apply Corollary 3.2 to rewrite $\nu$ and $d$ as functions of the unknown $\alpha: \nu(\alpha)$ and $d(\alpha)$.

Then, we consider the following equation:

$$
\frac{\partial c(x, t)}{\partial t}=-\nu(\alpha) \frac{\partial c(x, t)}{\partial x}+d(\alpha) \frac{\partial^{\alpha} c(x, t)}{\partial x^{\alpha}}+r(x, t) .
$$

If $\phi_{n}$, which is different than when $\phi_{m}$ is used in Proposition 3.2, is at least second order modulating function on $\left[0, L_{1}\right]$ where $n=M+1$, then using a similar way of obtaining (3.8), we get

$$
\begin{aligned}
\nu(\alpha) \int_{0}^{L_{1}}-\frac{\partial \phi_{n}\left(L_{1}-x\right)}{\partial x} c(x, t) \mathrm{d} x+d(\alpha) \int_{0}^{L_{1}} \frac{\partial^{\alpha} \phi_{n}(x)}{\partial x^{\alpha}} c\left(L_{1}-x, t\right) \mathrm{d} x \\
=\int_{0}^{L_{1}} \phi_{n}\left(L_{1}-x\right) \frac{\partial c(x, t)}{\partial t} \mathrm{~d} x-\int_{0}^{L_{1}} r(x, t) \phi_{n}\left(L_{1}-x\right) \mathrm{d} x .
\end{aligned}
$$

Since $\alpha$ is the only unknown in (4.2), we can write it as follows:

$$
K_{t}(\alpha)=U_{t},
$$

where

$$
K_{t}(\alpha):=d(\alpha) \int_{0}^{L_{1}} \frac{\partial^{\alpha} \phi_{n}(x)}{\partial x^{\alpha}} c\left(L_{1}-x, t\right) \mathrm{d} x-\nu(\alpha) \int_{0}^{L_{1}} \frac{\partial \phi_{n}\left(L_{1}-x\right)}{\partial x} c(x, t) \mathrm{d} x
$$

and

$$
U_{t}:=\int_{0}^{L_{1}} \phi_{n}\left(L_{1}-x\right)\left[\frac{\partial c(x, t)}{\partial t}-r(x, t)\right] \mathrm{d} x .
$$

Stage 2. In this stage, the inverse problem is formulated as a solution to a nonlinear equation with respect to the unknown $\alpha$,

$$
J(\alpha)=K_{T}(\alpha)-U_{T}=0,
$$

where $K_{T}(\alpha)$ and $U_{T}$ are computed using the measurements given in (1.3) at a final time. 
4.1.1. Newton's iteration approach. We propose using first order Newton's type method to solve the nonlinear equation given in (4.6). At each iteration, the order $\alpha$ is updated using

$$
K_{T}\left(\alpha_{k}\right)-U_{T}=-\Delta \alpha_{k} K_{T}^{\prime}\left(\alpha_{k}\right),
$$

where

$$
\alpha_{k+1}=\alpha_{k}+\Delta \alpha_{k}
$$

and the gradient $K^{\prime}(\alpha)$ is computed using the following proposition.

In Proposition 4.1, the derivatives $\nu^{\prime}(\alpha)$ and $d^{\prime}(\alpha)$ will be obtained using a system of linear equations, where the modulating functions used to obtain $\nu$ and $d$ in Corollary 3.2 must be used to obtain the corresponding $\nu^{\prime}(\alpha)$ and $d^{\prime}(\alpha)$.

Proposition 4.1. For $0<L_{1} \leq L$, let $\left\{\phi_{m}(x)\right\}_{m=1}^{M}$ be a set of $M$ modulating functions of at least order 2 defined on the interval $\left[0, L_{1}\right]$ where $2 \leq M$. Then the parameters $\nu$ and $d$ can be written in terms of $\alpha$ using Proposition 3.2, and the following linear system estimates the derivatives of $d$ and $\nu$ with respect to $\alpha$ :

$$
\mathbf{P}\left[\begin{array}{c}
\nu^{\prime}(\alpha) \\
d^{\prime}(\alpha)
\end{array}\right]=-d(\alpha) \mathbf{B}^{\prime}, \text { where } \mathbf{B}^{\prime}=\left[\begin{array}{c}
B_{1}^{\prime} \\
B_{2}^{\prime} \\
\vdots \\
B_{M}^{\prime}
\end{array}\right],
$$

with

$$
B_{m}^{\prime}=\frac{\partial}{\partial \alpha} B_{m}=\int_{0}^{L_{1}} \frac{\partial}{\partial \alpha} \frac{\partial^{\alpha} \phi_{m}(x)}{\partial x^{\alpha}} c\left(L_{1}-x, t\right) \mathrm{d} x,
$$

and $P, A_{m}$, and $B_{m}$ are defined as in (3.10), (3.11), and (3.12), respectively, for $m=1,2, \ldots, M$.

Proof. We first write the parameters $\nu$ and $d$ in terms of $\alpha$ using Proposition 3.2, then the proof can be obtained by differentiating (4.2) with respect to $\alpha$ where $\phi_{n}$ is replaced by $\phi_{m}$, which gives

$$
\begin{array}{r}
\nu^{\prime}(\alpha) \int_{0}^{L_{1}}-\frac{\partial \phi_{m}\left(L_{1}-x\right)}{\partial x} c(x, t) \mathrm{d} x+d^{\prime}(\alpha) \int_{0}^{L_{1}} \frac{\partial^{\alpha} \phi_{m}(x)}{\partial x^{\alpha}} c\left(L_{1}-x, t\right) \mathrm{d} x \\
+d(\alpha) \int_{0}^{L_{1}} \frac{\partial}{\partial \alpha} \frac{\partial^{\alpha} \phi_{m}(x)}{\partial x^{\alpha}} c\left(L_{1}-x, t\right) \mathrm{d} x=0,
\end{array}
$$

for $m=1,2, \ldots, M$.

Similarly as in Proposition 3.2, the estimates $\nu^{\prime}(\alpha)$ and $d^{\prime}(\alpha)$ can be obtained by solving the system given in (4.9) using the least square method. The derivative of $\frac{\partial^{\alpha} \phi_{m}(x)}{\partial x^{\alpha}}$ with respect to $\alpha$ can be computed using Proposition 2.4.

Now, using Proposition 4.1 we can derive the analytic form of $K^{\prime}(\alpha)$.

TheOREM 4.2. Using $\nu(\alpha)$ and $d(\alpha)$, which are the estimations given by Proposition 3.2, and $K(\alpha)$ as given in (4.4), the gradient $K^{\prime}(\alpha)$ exists and can be computed as follows:

$$
\begin{aligned}
K^{\prime}(\alpha)=d^{\prime}(\alpha) & \int_{0}^{L_{1}} \frac{\partial^{\alpha} \phi_{n}(x)}{\partial x^{\alpha}} c\left(L_{1}-x, t\right) \mathrm{d} x-\nu^{\prime}(\alpha) \int_{0}^{L_{1}} \frac{\partial \phi_{n}\left(L_{1}-x\right)}{\partial x} c(x, t) \mathrm{d} x \\
& +d(\alpha) \int_{0}^{L_{1}} \frac{\partial}{\partial \alpha} \frac{\partial^{\alpha} \phi_{n}(x)}{\partial x^{\alpha}} c\left(L_{1}-x, t\right) \mathrm{d} x,
\end{aligned}
$$

Copyright $\odot$ by SIAM. Unauthorized reproduction of this article is prohibited. 
where $n=M+1$ and $d^{\prime}(\alpha), \nu^{\prime}(\alpha)$ are given by Proposition 4.1 .

Proof. $K^{\prime}(\alpha)$ can be obtained directly by differentiating (4.4) with respect to $\alpha$. The differentiability of $K(\alpha)$ mainly depends on the differentiability of (2.1) with respect to $\alpha$, which always exists as the gamma function is differentiable, and thanks to $\mathrm{H} 2$.

Remark 4.1. Newton's iteration is a gradient based method, and we know that for any gradient based algorithm most of the computational effort is spent on computing the gradient at each step. However, here, thanks to the MFM, we have a closed form gradient. This analytical form is more stable and requires less computational power. Further, using a similar way, we can efficiently compute higher order derivatives.

Remark 4.2. In general, when using iterative methods to solve nonlinear equations, it is often difficult to establish the invertability of the gradient, which is the case here. However, from our experimental experiences it is shown that $K^{\prime}$ is nonsingular.

4.2. Two stage algorithm. The proposed algorithm is described in Figure 1.

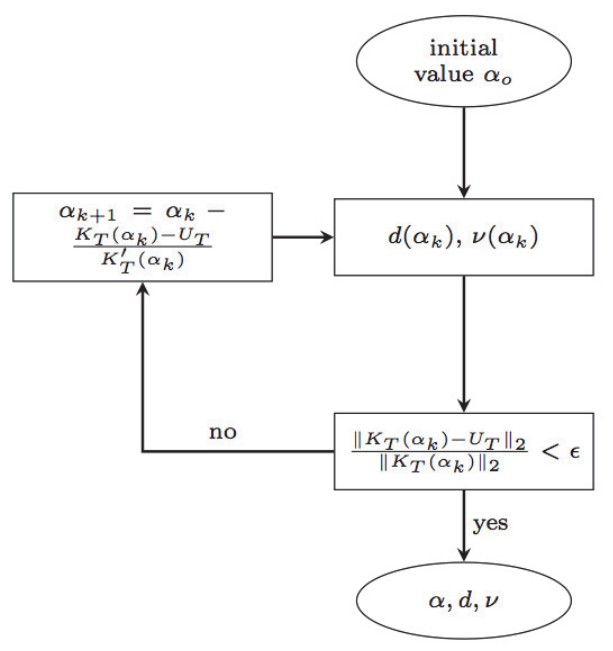

FIG. 1. Two stage algorithm to estimate $\nu, d$, and $\alpha$.

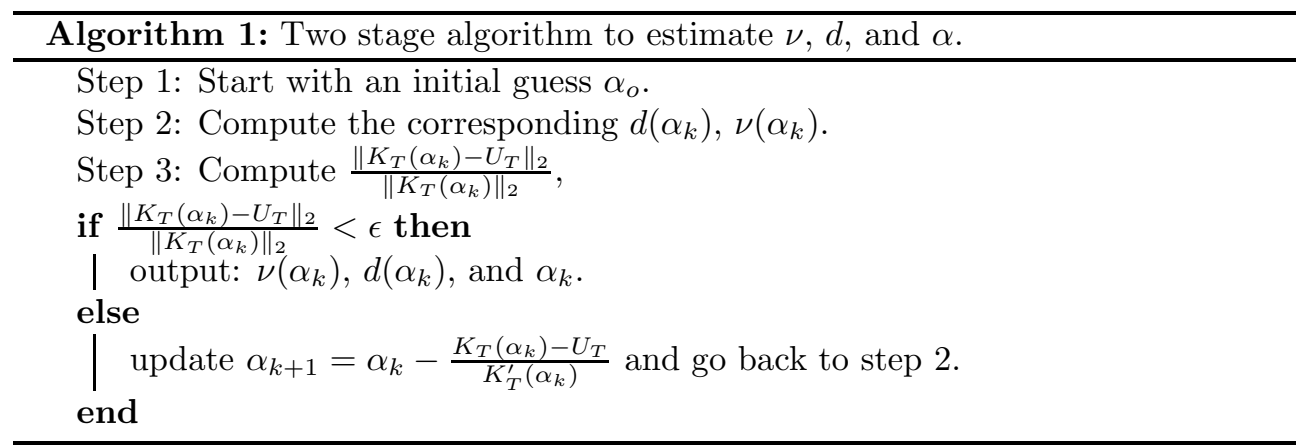

5. Convergence of the algorithm. Our goal is to analyze the convergence of the proposed two stage algorithm when the standard assumptions of the Newton's 
iteration algorithm hold. Without loss of generality and for sake of simplicity, we write the convergence proof for $M=2$.

In order to show the local convergence of the presented algorithm we make the following assumption:

H3. The functions $\frac{\partial^{\alpha} \phi(x)}{\partial x^{\alpha}}$ and $\frac{\partial}{\partial \alpha} \frac{\partial^{\alpha} \phi(x)}{\partial x^{\alpha}}$ are Lipschitz continuous in $\alpha$, i.e., there exist constants $\gamma_{1}$ and $\gamma_{2}$ such that, for all $\alpha_{1}$ and $\alpha_{2}$ in ]1,2],

$$
\left|\frac{\partial^{\alpha_{1}} \phi(x)}{\partial x^{\alpha_{1}}}-\frac{\partial^{\alpha_{2}} \phi(x)}{\partial x^{\alpha_{2}}}\right| \leq \gamma_{1}\left|\alpha_{1}-\alpha_{2}\right|
$$

and

$$
\left|\frac{\partial}{\partial \alpha_{1}} \frac{\partial^{\alpha_{1}} \phi(x)}{\partial x^{\alpha_{1}}}-\frac{\partial}{\partial \alpha_{2}} \frac{\partial^{\alpha_{2}} \phi(x)}{\partial x^{\alpha_{2}}}\right| \leq \gamma_{2}\left|\alpha_{1}-\alpha_{2}\right|
$$

Note that the inequalities given in (5.1) and (5.2) hold uniformly with respect to $x$.

Before obtaining the convergence of our proposed two stage algorithm, we first prove some important results.

Proposition 5.1. Under the assumptions H1-H3 the following hold: for $i=$ $1,2, \ldots, M+1$ and $j=1,2, \ldots, M+1$,

1. $A_{i}$ and $y_{i}$ defined as in (3.11) and (3.13), respectively, are bounded.

2. The function $B_{i}:[1,2] \rightarrow \mathbb{R}$ defined as in (3.12) is bounded.

3. Then function $B_{i}^{\prime}:[1,2] \rightarrow \mathbb{R}$ defined as in (4.10) is bounded.

4. The function $h_{i, j}$ defined as follows: for $\left.\left.\alpha \in\right] 1,2\right]$,

$$
h_{i, j}(\alpha)=A_{i} B_{j}(\alpha)-A_{j} B_{i}(\alpha)
$$

is bounded.

5. For $\alpha \in] 1,2], d(\alpha)$ given by (3.9) is bounded.

6. $B_{i}(\alpha)$ defined as in (3.12) is Lipschitz continuous in $\alpha$, that is, there exists a constant $\eta_{1}$ such that for all $\alpha_{1}$ and $\alpha_{2}$ in ]1,2],

$$
\left|B_{i}\left(\alpha_{1}\right)-B_{i}\left(\alpha_{2}\right)\right| \leq \eta_{1}\left|\alpha_{1}-\alpha_{2}\right| .
$$

7. $B_{i}^{\prime}(\alpha)$ defined as in (4.10) is Lipschitz continuous in $\alpha$, that is, there exists a constant $\eta_{2}$ such that for all $\alpha_{1}$ and $\alpha_{2}$ in ]1,2],

$$
\left|B_{i}^{\prime}\left(\alpha_{1}\right)-B_{i}^{\prime}\left(\alpha_{2}\right)\right| \leq \eta_{2}\left|\alpha_{1}-\alpha_{2}\right| .
$$

8. For $\alpha \in] 1,2]$ the following functions are Lipschitz continuous:

(a) $h_{i, j}(\alpha)$ defined as in (5.3).

(b) $\left[h_{i, j}(\alpha)\right]^{2}$.

9. $g(\alpha)$ defined by: for $\alpha \in] 1,2]$,

$$
g_{i, j}(\alpha)=\frac{B_{i}(\alpha) B_{j}^{\prime}(\alpha)}{h_{i, j}(\alpha)^{2}} .
$$

Proof. The proofs of 1-3 can be directly deduced from $\mathrm{H} 1$ and $\mathrm{H} 2$, respectively.

4. This can be directly deduced from H1 and the continuity of $\frac{\partial^{\alpha} \phi}{\partial x^{\alpha}}$.

5. This can be obtained directly using 1 and 4 . 
6. Let $\left.\left.\alpha_{1}, \alpha_{2} \in\right] 1,2\right]$, then

$$
\begin{gathered}
\left|B_{i}\left(\alpha_{1}\right)-B_{i}\left(\alpha_{2}\right)\right| \\
=\left|\int_{0}^{L_{1}} \frac{\partial^{\alpha_{1}} \phi_{i}(x)}{\partial x^{\alpha_{1}}} c\left(L_{1}-x, t\right) d x-\int_{0}^{L_{1}} \frac{\partial^{\alpha_{2}} \phi_{i}(x)}{\partial x^{\alpha_{2}}} c\left(L_{1}-x, t\right) d x\right| \\
\leq\left|\frac{\partial^{\alpha_{1}} \phi_{i}(x)}{\partial x^{\alpha_{1}}}-\frac{\partial^{\alpha_{2}} \phi_{i}(x)}{\partial x^{\alpha_{2}}}\right|\left|c\left(L_{1}-x, t\right)\right| .
\end{gathered}
$$

By the continuity of $c(\cdot, T)$ and H3, there exist constants $M_{c}$ and $\gamma_{1}$ such that

$$
\left|B_{i}\left(\alpha_{1}\right)-B_{i}\left(\alpha_{2}\right)\right| \leq \gamma_{1} M_{c}\left|\alpha_{1}-\alpha_{2}\right|,
$$

and setting $\eta_{1}=\gamma_{1} M_{c}$, we get inequality (5.4).

7. The proof can be obtained similarly as in 6 .

8. (a) The result can be obtained directly by applying 1 and 6 .

(b) For $\left.\left.\alpha_{1}, \alpha_{2} \in\right] 1,2\right]$, we get

$$
\begin{aligned}
\left|\left[h_{i, j}\left(\alpha_{1}\right)\right]^{2}-\left[h_{i, j}\left(\alpha_{2}\right)\right]^{2}\right|= & \mid A_{i}^{2}\left[B_{j}^{2}\left(\alpha_{1}\right)-B_{j}^{2}\left(\alpha_{2}\right)\right]+A_{j}^{2}\left[B_{i}^{2}\left(\alpha_{1}\right)-B_{i}^{2}\left(\alpha_{2}\right)\right] \\
& +2 A_{i} A_{j}\left[B_{j}\left(\alpha_{2}\right) B_{i}\left(\alpha_{2}\right)-B_{j}\left(\alpha_{1}\right) B_{i}\left(\alpha_{1}\right)\right] \mid .
\end{aligned}
$$

Now, by adding and subtracting $B_{i}\left(\alpha_{2}\right) B_{j}\left(\alpha_{1}\right)$ from the last term of (5.10) and applying the triangular inequality, we obtain

$$
\begin{aligned}
\left|\left[h_{i, j}\left(\alpha_{1}\right)\right]^{2}-\left[h_{i, j}\left(\alpha_{2}\right)\right]^{2}\right| \leq & \left|A_{i}^{2}\left[B_{j}\left(\alpha_{1}\right)+B_{j}\left(\alpha_{2}\right)\right]\right|\left|B_{j}\left(\alpha_{1}\right)-B_{j}\left(\alpha_{2}\right)\right| \\
& +\left|A_{j}^{2}\left[B_{i}\left(\alpha_{1}\right)+B_{i}\left(\alpha_{2}\right)\right]\right|\left|B_{i}\left(\alpha_{1}\right)-B_{i}\left(\alpha_{2}\right)\right| \\
& +\left|2 A_{i} A_{j} B_{i}\left(\alpha_{2}\right)\right|\left|B_{j}\left(\alpha_{2}\right)-B_{j}\left(\alpha_{1}\right)\right| \\
& +\left|2 A_{i} A_{j} B_{j}\left(\alpha_{1}\right)\right|\left|B_{i}\left(\alpha_{2}\right)-B_{i}\left(\alpha_{1}\right)\right| .
\end{aligned}
$$

Finally, by 1,2 , and 6 there exist constants $M_{a}, M_{b}$, and $\eta_{1}$ such that

$$
\left|\left[h_{i, j}\left(\alpha_{1}\right)\right]^{2}-\left[h_{i, j}\left(\alpha_{2}\right)\right]^{2}\right| \leq 8 M_{a}^{2} M_{b} \eta_{1}\left|\alpha_{1}-\alpha_{2}\right|,
$$

where $M_{b}=\max \left\{M_{b_{i}}, M_{b_{j}}\right\}$ and $M_{a}=\max \left\{M_{a_{i}}, M_{a_{j}}\right\}$ for $\left|B_{i}\right| \leq$ $M_{b_{i}} \leq M_{b},\left|B_{j}\right| \leq M_{b_{j}} \leq M_{b}$, and $\left|A_{i}\right| \leq M_{a_{i}},\left|A_{i}\right| \leq M_{a_{i}} \leq M_{a}$.

9. Let $\left.\left.\alpha_{1}, \alpha_{2} \in\right] 1,2\right]$, then

$$
=\left|\frac{1}{\left[h_{i, j}\left(\alpha_{1}\right) h_{i, j}\left(\alpha_{2}\right)\right]^{2}}\right|\left|h_{i, j}\left(\alpha_{2}\right)^{2} B_{i}\left(\alpha_{1}\right) B_{j}^{\prime}\left(\alpha_{1}\right)-h_{i, j}\left(\alpha_{1}\right)^{2} B_{i}\left(\alpha_{2}\right) B_{j}^{\prime}\left(\alpha_{2}\right)\right| .
$$

Now, adding and subtracting $h_{i, j}\left(\alpha_{2}\right)^{2} B_{i}\left(\alpha_{2}\right) B_{j}^{\prime}\left(\alpha_{1}\right)$ and $h_{i, j}\left(\alpha_{1}\right)^{2} B_{i}\left(\alpha_{1}\right) B_{j}^{\prime}\left(\alpha_{2}\right)$ from the last term of (5.13) and by applying the triangular inequality, we have

$$
\begin{aligned}
\left|g_{i, j}\left(\alpha_{1}\right)-g_{i, j}\left(\alpha_{2}\right)\right| \leq & \left|\frac{1}{\left[h_{i, j}\left(\alpha_{1}\right) h_{i, j}\left(\alpha_{2}\right)\right]^{2}}\right| \\
& \left|\left[h_{i, j}\left(\alpha_{2}\right)^{2} B_{j}^{\prime}\left(\alpha_{1}\right)+h_{i, j}\left(\alpha_{1}\right)^{2} B_{j}^{\prime}\left(\alpha_{2}\right)\right]\left[B_{i}\left(\alpha_{1}\right)-B_{i}\left(\alpha_{2}\right)\right]\right| \\
& +\left|h_{i, j}\left(\alpha_{2}\right)^{2} B_{i}\left(\alpha_{2}\right) B_{j}^{\prime}\left(\alpha_{1}\right)-h_{i, j}\left(\alpha_{1}\right)^{2} B_{i}\left(\alpha_{1}\right) B_{j}^{\prime}\left(\alpha_{2}\right)\right|
\end{aligned}
$$

Copyright (c) by SIAM. Unauthorized reproduction of this article is prohibited. 
by adding and subtracting $h_{i, j}\left(\alpha_{2}\right)^{2} B_{i}\left(\alpha_{2}\right) B_{j}^{\prime}\left(\alpha_{2}\right)$ of the last term, we get

$$
\begin{aligned}
\left|g_{i, j}\left(\alpha_{1}\right)-g_{i, j}\left(\alpha_{2}\right)\right| \leq & \left|\frac{1}{\left[h_{i, j}\left(\alpha_{1}\right) h_{i, j}\left(\alpha_{2}\right)\right]^{2}}\right| \\
\cdot & \begin{aligned}
\mid & \left.h_{i, j}\left(\alpha_{2}\right)^{2} B_{j}^{\prime}\left(\alpha_{1}\right)+h_{i, j}\left(\alpha_{1}\right)^{2} B_{j}^{\prime}\left(\alpha_{2}\right)\right]\left[B_{i}\left(\alpha_{1}\right)-B_{i}\left(\alpha_{2}\right)\right] \mid \\
& +\mid h_{i, j}\left(\alpha_{2}\right)^{2} B_{i}\left(\alpha_{2}\right)\left[B_{j}^{\prime}\left(\alpha_{1}\right)-B_{j}^{\prime}\left(\alpha_{2}\right)\right] \\
& \quad+B_{j}^{\prime}\left(\alpha_{2}\right)\left[h_{i, j}\left(\alpha_{2}\right)^{2} B_{i}\left(\alpha_{2}\right)-h_{i, j}\left(\alpha_{1}\right)^{2} B_{i}\left(\alpha_{1}\right)\right] \mid,
\end{aligned}
\end{aligned}
$$

and by adding and subtracting $h_{i, j}\left(\alpha_{2}\right)^{2} B_{i}\left(\alpha_{1}\right)$ of the last term, we obtain

$$
\begin{aligned}
\left|g_{i, j}\left(\alpha_{1}\right)-g_{i, j}\left(\alpha_{2}\right)\right| & \leq\left|\frac{1}{\left.h_{i, j}\left(\alpha_{1}\right) h_{i, j}\left(\alpha_{2}\right)\right]^{2}}\right| \\
& \cdot\left[\mid\left[h_{i, j}\left(\alpha_{2}\right)^{2} B_{j}^{\prime}\left(\alpha_{1}\right)+h_{i, j}\left(\alpha_{1}\right)^{2} B_{j}^{\prime}\left(\alpha_{2}\right)|| B_{i}\left(\alpha_{1}\right)-B_{i}\left(\alpha_{2}\right) \mid\right.\right. \\
& +\left|h_{i, j}\left(\alpha_{2}\right)^{2} B_{i}\left(\alpha_{2}\right)\right|\left|B_{j}^{\prime}\left(\alpha_{1}\right)-B_{j}^{\prime}\left(\alpha_{2}\right)\right| \\
& +\left|B_{j}^{\prime}\left(\alpha_{2}\right) h_{i, j}\left(\alpha_{2}\right)^{2}\right|\left|B_{i}\left(\alpha_{2}\right)-B_{i}\left(\alpha_{1}\right)\right| \\
& \left.+\left|B_{i}\left(\alpha_{1}\right)\right|\left|h_{i, j}\left(\alpha_{2}\right)^{2}-h_{i, j}\left(\alpha_{1}\right)^{2}\right|\right] .
\end{aligned}
$$

Finally, by 2, 3, 4 and by applying 6,7 , and $8 \mathrm{~b}$, there exist constants $M_{b}$, $M_{b}^{\prime}, M_{\alpha}, \eta_{1}, \eta_{2}$, and $\eta_{3}$, respectively, such that

$$
\left|g_{i, j}\left(\alpha_{1}\right)-g_{i, j}\left(\alpha_{2}\right)\right| \leq M_{g}\left|\alpha_{1}-\alpha_{2}\right|,
$$

where $M_{g}=\left[3 M_{\alpha}^{2} M_{b}^{\prime} \eta_{1}+M_{\alpha}^{2} M_{b} \eta_{2}+M_{b} \eta_{3}\right]$. 71].

To prove the convergence, we recall the following theorem referred to in [11, p.

THEOREM 5.2 (see [11]). If the following assumptions hold:

$S 1$. The equation $J(\alpha)=0$ has a solution $\alpha^{*}$,

$S 2$. $J^{\prime}:[1,2] \rightarrow \mathbb{R}$ is Lipschitz continuous with constant $\gamma$,

S3. $J^{\prime}\left(\alpha^{*}\right)$ is nonsingular,

then there exists $\delta$ such that if $\alpha_{0} \in B(\delta)$, the Newton iteration

$$
\alpha_{k+1}=\alpha_{k}-\frac{J\left(\alpha_{k}\right)}{J^{\prime}\left(\alpha_{k}\right)}
$$

converges q-quardatically to $\alpha *$.

Now, we are ready to prove the main result in this section.

THEOREM 5.3. Assume that the solution of $J(\alpha)=0$, where $J(\alpha)$ is defined as in (4.6), exists and $J^{\prime}$ is nonsingular. Then, under the assumptions $\mathrm{H} 1-\mathrm{H} 3$ and for $M=2$, the proposed two stage algorithm given in Algorithm 1 converges locally.

Proof. Since we assume that $J(\alpha)$ has a solution and $J^{\prime}\left(\alpha^{*}\right)$ is nonsignular, then if $J^{\prime}$ defined in section 4.1.1 is Lipschitz continuous, the convergence result of the Newton method holds [11].

Recall that

$$
J^{\prime}(\alpha)=K^{\prime}(\alpha)=A_{n} v^{\prime}(\alpha)+B_{n}(\alpha) d^{\prime}(\alpha)+B_{n}^{\prime}(\alpha) d(\alpha),
$$

Copyright (c) by SIAM. Unauthorized reproduction of this article is prohibited. 
where $A_{n}, B_{n}(\alpha)$, and $B_{n}^{\prime}(\alpha)$ are defined as in (3.11), (3.12), and (4.10), respectively, and $n=M+1$.

Using Proposition 3.2, we have

$$
d(\alpha)=\frac{1}{h_{m_{1}, m_{2}}(\alpha)}\left[-A_{m_{2}} C_{m 1}+A_{m_{1}} C_{m 2}\right]
$$

and from Proposition 4.1, we have

$$
\left[\begin{array}{c}
\nu^{\prime}(\alpha) \\
d^{\prime}(\alpha)
\end{array}\right]=\frac{d(\alpha)}{h_{m_{1}, m_{2}}(\alpha)}\left[\begin{array}{c}
B_{m_{2}}(\alpha) B_{m 1}^{\prime}(\alpha)-B_{m_{1}}(\alpha) B_{m 2}^{\prime}(\alpha) \\
-A_{m_{2}} B_{m 1}^{\prime}(\alpha)+A_{m_{1}} B_{m 2}^{\prime}(\alpha)
\end{array}\right]
$$

where $h(\alpha)$ is defined in (5.3).

Now, we can show that $J^{\prime}$ is Lipschitz continuous. Let $\left.\left.\alpha_{1}, \alpha_{2} \in\right] 1,2\right]$, then

$$
\begin{aligned}
& \left|J^{\prime}\left(\alpha_{1}\right)-J^{\prime}\left(\alpha_{2}\right)\right|=\mid A_{n} v^{\prime}\left(\alpha_{1}\right) \\
& \quad+B_{n}\left(\alpha_{1}\right) d^{\prime}\left(\alpha_{1}\right)+B_{n}^{\prime}\left(\alpha_{1}\right) d\left(\alpha_{1}\right)-A_{n} v^{\prime}\left(\alpha_{2}\right)-B_{n}\left(\alpha_{2}\right) d^{\prime}\left(\alpha_{2}\right)-B_{n}^{\prime}\left(\alpha_{1}\right) d\left(\alpha_{2}\right) \mid .
\end{aligned}
$$

Substituting the values of $\nu^{\prime}(\alpha)$ and $d^{\prime}(\alpha)$ given in (5.21) into (5.22), we get

$$
\begin{aligned}
\left|J^{\prime}\left(\alpha_{1}\right)-J^{\prime}\left(\alpha_{2}\right)\right|= & \mid A_{n} d\left(\alpha_{1}\right) \frac{B_{m_{2}}\left(\alpha_{1}\right) B_{m 1}^{\prime}\left(\alpha_{1}\right)-B_{m_{1}}\left(\alpha_{1}\right) B_{m 2}^{\prime}\left(\alpha_{1}\right)}{h_{m_{1}, m_{2}}\left(\alpha_{1}\right)} \\
& +B_{n}\left(\alpha_{1}\right) d\left(\alpha_{1}\right) \frac{-A_{m_{2}} B_{m 1}^{\prime}\left(\alpha_{1}\right)+A_{m_{1}} B_{m 2}^{\prime}\left(\alpha_{1}\right)}{h_{m_{1}, m_{2}}\left(\alpha_{1}\right)}+B_{n}^{\prime}\left(\alpha_{1}\right) d\left(\alpha_{1}\right) \\
& -A_{n} d\left(\alpha_{2}\right) \frac{B_{m_{2}}\left(\alpha_{2}\right) B_{m 1}^{\prime}\left(\alpha_{2}\right)-B_{m_{1}}\left(\alpha_{2}\right) B_{m 2}^{\prime}\left(\alpha_{2}\right)}{h_{m_{1}, m_{2}}\left(\alpha_{2}\right)} \\
& -B_{n}\left(\alpha_{2}\right) d\left(\alpha_{2}\right) \frac{-A_{m_{2}} B_{m 1}^{\prime}\left(\alpha_{2}\right)+A_{m_{1}} B_{m 2}^{\prime}\left(\alpha_{2}\right)}{h_{m_{1}, m_{2}}\left(\alpha_{2}\right)}-B_{n}^{\prime}\left(\alpha_{2}\right) d\left(\alpha_{2}\right) \mid
\end{aligned}
$$

$$
\begin{aligned}
& =\mid A_{n}\left[d\left(\alpha_{1}\right) \frac{B_{m_{2}}\left(\alpha_{1}\right) B_{m 1}^{\prime}\left(\alpha_{1}\right)}{h_{m_{1}, m_{2}}\left(\alpha_{1}\right)}-d\left(\alpha_{2}\right) \frac{B_{m_{2}}\left(\alpha_{2}\right) B_{m 1}^{\prime}\left(\alpha_{2}\right)}{h_{m_{1}, m_{2}}\left(\alpha_{2}\right)}\right] \\
& -A_{n}\left[d\left(\alpha_{1}\right) \frac{B_{m_{1}}\left(\alpha_{1}\right) B_{m 2}^{\prime}\left(\alpha_{1}\right)}{h_{m_{1}, m_{2}}\left(\alpha_{1}\right)}-d\left(\alpha_{2}\right) \frac{B_{m_{1}}\left(\alpha_{2}\right) B_{m 2}^{\prime}\left(\alpha_{2}\right)}{h_{m_{1}, m_{2}}\left(\alpha_{2}\right)}\right] \\
& +A_{m_{2}}\left[\frac{-d\left(\alpha_{1}\right) B_{n}\left(\alpha_{1}\right) B_{m 1}^{\prime}\left(\alpha_{1}\right)}{h_{m_{1}, m_{2}}\left(\alpha_{1}\right)}+d\left(\alpha_{2}\right) \frac{B_{n}\left(\alpha_{2}\right) B_{m 1}^{\prime}(\alpha 2)}{h_{m_{1}, m_{2}}\left(\alpha_{2}\right)}\right] \\
& +A_{m_{1}}\left[d\left(\alpha_{1}\right) \frac{B_{n}\left(\alpha_{1}\right) B_{m 2}^{\prime}\left(\alpha_{1}\right)}{h_{m_{1}, m_{2}}\left(\alpha_{1}\right)}-d\left(\alpha_{2}\right) \frac{B_{n}\left(\alpha_{2}\right) B_{m 2}^{\prime}\left(\alpha_{2}\right)}{h_{m_{1}, m_{2}}\left(\alpha_{2}\right)}\right] \\
& +B_{n}^{\prime}\left(\alpha_{1}\right) d\left(\alpha_{1}\right)-B_{n}^{\prime}\left(\alpha_{2}\right) d\left(\alpha_{2}\right) \mid .
\end{aligned}
$$

Copyright $@$ by SIAM. Unauthorized reproduction of this article is prohibited. 
Next, substitute $d\left(\alpha_{i}\right)$ for $i=1,2$ by (5.20), then (5.24) becomes

$$
\begin{aligned}
\left|J^{\prime}\left(\alpha_{1}\right)-J^{\prime}\left(\alpha_{2}\right)\right|= & \left|-A_{m 2} C_{m 1}+A_{m 1} C_{m 2}\right| \\
& \cdot \mid A_{n}\left[g_{m_{2}, m_{1}}\left(\alpha_{1}\right)-g_{m_{2}, m_{1}}\left(\alpha_{2}\right)\right]-A_{n}\left[g_{m_{1}, m_{2}}\left(\alpha_{1}\right)-g_{m_{1}, m_{2}}\left(\alpha_{2}\right)\right] \\
& -A_{m_{2}}\left[g_{n, m_{1}}\left(\alpha_{1}\right)-g_{n, m 1}\left(\alpha_{2}\right)\right]+A_{m_{1}}\left[g_{n, m_{2}}\left(\alpha_{1}\right)-g_{n, m_{2}}\left(\alpha_{2}\right)\right] \\
& +\frac{1}{h_{m_{1}, m_{2}}\left(\alpha_{1}\right) h_{m_{1}, m_{2}}\left(\alpha_{2}\right)}\left[h_{m_{1}, m_{2}}\left(\alpha_{2}\right) B_{n}^{\prime}\left(\alpha_{1}\right)-h_{m_{1}, m_{2}}\left(\alpha_{1}\right) B_{n}^{\prime}\left(\alpha_{2}\right)\right] \mid,
\end{aligned}
$$

where $g_{i, j}(\alpha)$ is defined as in (5.6).

Adding and subtracting $h_{m_{1}, m_{2}}\left(\alpha_{2}\right) B_{n}^{\prime}\left(\alpha_{2}\right)$ of the last term of (5.25) and applying the triangular inequality, we have

$$
\begin{aligned}
\left|J^{\prime}\left(\alpha_{1}\right)-J^{\prime}\left(\alpha_{2}\right)\right| \leq & \left|-A_{m 2} C_{m 1}+A_{m 1} C_{m 2}\right| \\
& \cdot\left[\left|A_{n}\right|\left|g_{m_{2}, m_{1}}\left(\alpha_{1}\right)-g_{m_{2}, m_{1}}\left(\alpha_{2}\right)\right|+\left|A_{n}\right|\left|g_{m_{1}, m_{2}}\left(\alpha_{1}\right)-g_{m_{1}, m_{2}}\left(\alpha_{2}\right)\right|\right. \\
& +\left|A_{m_{2}}\right|\left|g_{n, m_{1}}\left(\alpha_{1}\right)-g_{n, m 1}\left(\alpha_{2}\right)\right|+\left|A_{m_{1}}\right|\left|g_{n, m_{2}}\left(\alpha_{1}\right)-g_{n, m_{2}}\left(\alpha_{2}\right)\right| \\
& +\left|\frac{1}{h_{m_{1}, m_{2}}\left(\alpha_{1}\right)}\right|\left|B_{n}^{\prime}\left(\alpha_{1}\right)-B_{n}^{\prime}\left(\alpha_{2}\right)\right| \\
& \left.+\left|\frac{B_{n}^{\prime}\left(\alpha_{2}\right)}{h_{m_{1}, m_{2}}\left(\alpha_{1}\right) h_{m_{1}, m_{2}}\left(\alpha_{2}\right)}\right|\left|h_{m_{1}, m_{2}}\left(\alpha_{2}\right)-h_{m_{1}, m_{2}}\left(\alpha_{1}\right)\right|\right] .
\end{aligned}
$$

Finally the proof can be completed using 1,3, and 4 and by applying 8a, 7, and 9 of Proposition 5.1.

Remark 5.1. Note that in the previous theorem the local convergence was proved for $M=2$. However, using Proposition 5.1 the local convergence can easily be proved for $M>2$, where $d(\alpha), d^{\prime}(\alpha)$, and $\nu(\alpha)$ can be obtained using the least square method and $h(\alpha)=\sum_{i=1}^{M} A_{i}^{2} \sum_{i=1}^{M} B_{i}^{2}(\alpha)-\left(\sum_{i=1}^{M} A_{i} B_{i}(\alpha)\right)^{2}$.

6. Numerical results. In this section, we present some numerical results to show the efficiency and robustness of the presented method.

The choice of the modulating function is important since it affects the efficiency of the algorithm. In this section, we propose using polynomial modulating functions that satisfy $\mathrm{H} 1-\mathrm{H} 3$, and for which the fractional derivatives are easy to calculate, of the following form: for $a, b \in \mathbb{N}^{*}$ and $L_{1} \in \mathbb{R}$,

$$
\phi(x)=x^{a}\left(L_{1}-x\right)^{b}=\sum_{k=0}^{b} c_{k} x^{a+k},
$$

whose fractional derivative is known analytically and given by

$$
\frac{\partial^{\alpha} \phi(x)}{\partial x^{\alpha}}=\sum_{k=0}^{b} c_{k} \frac{\Gamma(a+k+1)}{\Gamma(a+k+1-\alpha)} x^{a+k-\alpha},
$$

Copyright $@$ by SIAM. Unauthorized reproduction of this article is prohibited. 
where $c_{k}=\left(\begin{array}{l}b \\ k\end{array}\right)(-1)^{k} L_{1}^{b-k}$ and the derivative of (6.2) with respect to $\alpha$ can be computed as follows:

$$
\frac{\partial}{\partial \alpha} \frac{\partial^{\alpha} \phi(x)}{\partial x^{\alpha}}=-\frac{\partial^{\alpha} \phi(x)}{\partial x^{\alpha}}\left[\ln (x)-\psi_{0}(n-\alpha)\right], \quad n-1 \leq \alpha<n,
$$

where $\psi_{0}(n-\alpha)=\frac{\Gamma^{\prime}(n-\alpha)}{\Gamma(n-\alpha)}$.

First, the coefficients $\nu$ and $d$ are estimated by solving the system given in (3.2). Then, we use the algorithm given in section 5 to estimate the parameters $\nu, d$, and $\alpha$ on a finite interval from noisy measurements. The value of $t$ is taken at the time $T$ where we have measurements. We consider the following polynomial modulating functions whose fractional derivatives are simple to calculate: $\phi_{m}(x)=x^{M+b+1-m}\left(L_{1}-x\right)^{b+m}$, where $L_{1} \leq L, m=1,2, \ldots, M$, and $M$ is the number of modulating functions. The derivatives with respect to $\alpha$ of $\frac{\partial^{\alpha} \phi_{m}(x)}{\partial x^{\alpha}}$ were computed analytically. Moreover, we apply the trapezoidal rule to numerically approximate the integrals with grid spacing $\Delta x$.

Example 6.1. We consider (1.1) with initial and Dirichlet boundary conditions given in (1.2), where $g(x)=x(L-x)$, and

$$
r(x, t)=\left\{\begin{array}{cl}
\cos (-t)\left[\nu(x)(L-2 x)-d(x)\left(\frac{L \Gamma(2)}{\Gamma(2-\alpha)} x^{1-\alpha}-\frac{\Gamma(3)}{\Gamma(3-\alpha)} x^{2-\alpha}\right)\right] & \\
\quad+\sin (-t) x(L-x), & 0<x<L, \\
0, & x=0, L .
\end{array}\right.
$$

The exact solution of the forward problem is $c(x, t)=\cos (-t) x(L-x)$ and the flux is $\frac{\partial c(x, t)}{\partial t}=\sin (-t) x(L-x)$.

Estimating the coefficients $\nu(x), d(x)$ when $\alpha$ is known. In this part, we assume that the differentiation order $\alpha$ is known and we estimate the variable coefficients $\nu(x)$ and $d(x)$ simultaneously, using the measurements given in (1.3). We set the differentiation order $\alpha=1.8$, the final time $T=1, b=1$, and the set of polynomial basis functions $\left\{1, x, x^{2}, x^{3}, \ldots\right\}$ is selected.

Noise free case. In Figures 2 and 3 , we have the estimated $\nu(x)=2(\sin (\pi x)+1)$ and $d(x)=0.7 x$ when using noise free measurements, where the length of the integration interval $L=2$. The results are reasonable for different number of modulating functions. This is confirmed in Table 1, where the errors of the estimated $\nu$ and $d$ are less than $1 \%$.

Noisy case. In Figures 4 and 5 , we can see the estimated $\nu(x)$ and $d(x)$ when $\nu(x)=2(\sin (\pi x)+1)$ and $d(x)=0.7 x$, where we add a $2 \%$ white Gaussian noise to the measurements. The results are satisfactory even with different numbers of modulating functions. This is confirmed in Tables 2 and 3 where the relative errors of the estimated $\nu$ and $d$ are reasonable even when adding $5 \%$ noise to both measurements.

In Figures 6 and 7, we can see the estimated $\nu(x)$ and $d(x)$ when $\nu(x)=2(\sin (\pi x)+$ 1) and $d(x)=5 \exp (-4 x)$, where we add a $2 \%$ white Gaussian noise to the measurements. In Table 4, we have the relative errors with different numbers of modulating functions.

Estimating constant coefficients $\nu, d$ when $\alpha$ is known. In this part, we estimate constant coefficients $\nu$ and $d$ when the differentiation order $\alpha$ is known. We set the average velocity $\nu=0.4$, the dispersion coefficient $d=1.1$, the differentiation order $\alpha=1.8$, and $\Delta x=\frac{1}{2100}$. Three modulating functions are used and the length of the integration interval $\left[0, L_{1}\right]$ has been increased. 


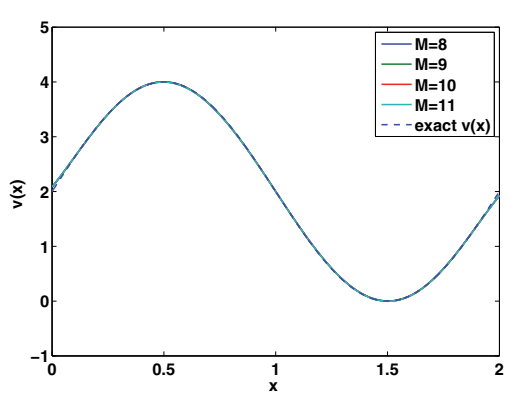

FIG. 2. Exact and estimated $\nu$ with different numbers of modulating functions when $\nu(x)=2(\sin (\pi x)+1), d(x)=0.7 x$, and $\Delta x=\frac{1}{2100}$.

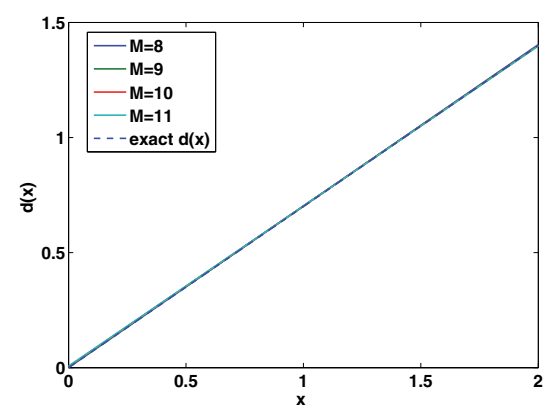

FIG. 3. Exact and estimated $d$ with different numbers of modulating functions when $\nu(x)=2(\sin (\pi x)+1), d(x)=0.7 x$, and $\Delta x=\frac{1}{2100}$.

TABLE 1

Relative errors of $\nu$ and $d$, where $\nu(x)=2(\sin (\pi x)+1), d(x)=0.7 x, \alpha=1.8$, and $\Delta x=\frac{1}{2100}$ in noise free case.

\begin{tabular}{ccc}
\hline \hline $\begin{array}{c}\text { Number of } \\
\text { modulating functions }\end{array}$ & $\begin{array}{c}\text { Relative errors } \\
\frac{\|\nu-\tilde{\nu}\|_{2}}{\|\nu\|_{2}}\end{array}$ & $\frac{\|d-\tilde{d}\|_{2}}{\|d\|_{2}}$ \\
\hline 8 & $0.58 \mathrm{E}-2$ & $0.25 \mathrm{E}-2$ \\
9 & $0.60 \mathrm{E}-2$ & $0.25 \mathrm{E}-2$ \\
10 & $0.61 \mathrm{E}-2$ & $0.30 \mathrm{E}-2$ \\
11 & $0.62 \mathrm{E}-2$ & $0.41 \mathrm{E}-2$ \\
\hline
\end{tabular}

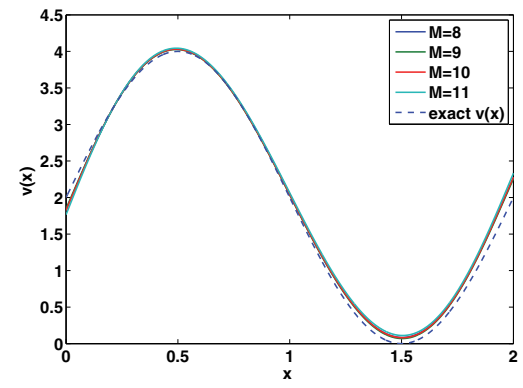

FIG. 4. Exact and estimated $\nu$ with different numbers of modulating functions when $\nu(x)=2(\sin (\pi x)+1), d(x)=0.7 x$ with $2 \%$ noise where $L_{1}=2$, and $\Delta x=\frac{1}{2100}$.

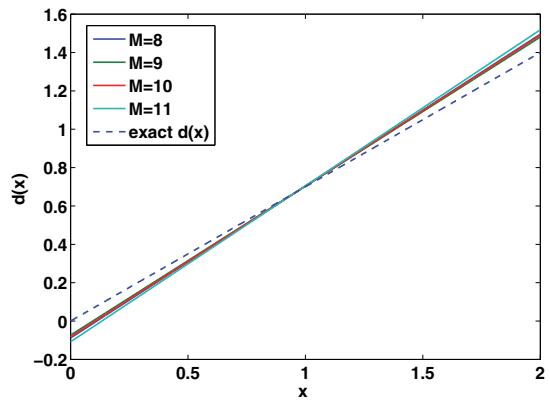

FIG. 5. Exact and estimated d with different numbers of modulating functions when $\nu(x)=2(\sin (\pi x)+1), d(x)=0.7 x$ with $2 \%$ noise where $L_{1}=2$, and $\Delta x=\frac{1}{2100}$.

TABle 2

Relative errors of $\nu=\frac{\|\nu-\tilde{\nu}\|_{2}}{\|\nu\|_{2}}$, where $\nu(x)=2(\sin (\pi x)+1), d(x)=0.7 x, \alpha=1.8$, and $\Delta x=$ $\frac{1}{2100}$ with different noise levels.

\begin{tabular}{cccc}
\hline \hline $\begin{array}{c}\text { Number of } \\
\text { modulating functions }\end{array}$ & $1 \%$ & $3 \%$ & $5 \%$ \\
\hline 8 & $1.82 \mathrm{E}-2$ & $5.56 \mathrm{E}-2$ & $8.88 \mathrm{E}-2$ \\
9 & $1.62 \mathrm{E}-2$ & $4.89 \mathrm{E}-2$ & $7.66 \mathrm{E}-2$ \\
10 & $1.89 \mathrm{E}-2$ & $5.34 \mathrm{E}-2$ & $8.13 \mathrm{E}-2$ \\
11 & $2.43 \mathrm{E}-2$ & $6.65 \mathrm{E}-2$ & $9.79 \mathrm{E}-2$ \\
\hline
\end{tabular}

Copyright (C) by SIAM. Unauthorized reproduction of this article is prohibited. 
TABLE 3

Relative errors of $d(x)=\frac{\|d-\tilde{d}\|_{2}}{\|d\|_{2}}$, where $\nu(x)=2(\sin (\pi x)+1), d(x)=0.7 x, \alpha=1.8$, and $\Delta x=\frac{1}{2100}$ with different noise levels.

\begin{tabular}{cccc}
\hline \hline $\begin{array}{c}\text { Number of } \\
\text { modulating functions }\end{array}$ & $1 \%$ & Noise level \\
\hline 8 & $3.35 \mathrm{E}-2$ & $9.23 \mathrm{E}-2$ & $13.74 \mathrm{E}-2$ \\
9 & $2.99 \mathrm{E}-2$ & $7.69 \mathrm{E}-2$ & $10.94 \mathrm{E}-2$ \\
10 & $3.39 \mathrm{E}-2$ & $8.47 \mathrm{E}-2$ & $11.66 \mathrm{E}-2$ \\
11 & $4.44 \mathrm{E}-2$ & $10.97 \mathrm{E}-2$ & $14.70 \mathrm{E}-2$ \\
\hline
\end{tabular}

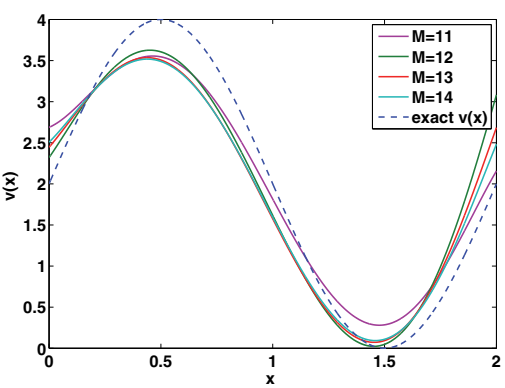

FIG. 6. Exact and estimated $\nu$ with different numbers of modulating functions when $\nu(x)=2(\sin (\pi x)+1)$ and $d(x)=5 \exp (-4 x)$ with $2 \%$ noise where $L_{1}=2$ and $\Delta x=\frac{1}{2100}$.

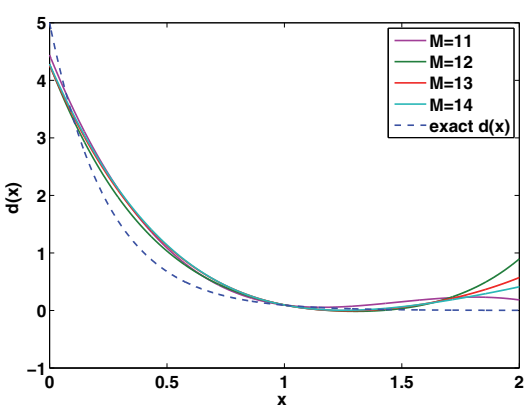

FIG. 7. Exact and estimated $d$ with different numbers of modulating functions when $\nu(x)=2(\sin (\pi x)+1)$ and $d(x)=5 \exp (-4 x)$ with $2 \%$ noise where $L_{1}=2$ and $\Delta x=\frac{1}{2100}$.

Noisy case. In Figure 8, we can see the estimated values of $\nu$ and $d$ when adding a $3 \%$ white Gaussian noise to the measurements. From Figure 9, we observe that the numerical results are quite satisfactory, where the relative error is less than $5 \%$ when integrating over the interval $[0,4.5]$ and drops to less than $1 \%$ as we increase the length of the integration interval. Although not presented, we would like to note that the results obtained using up to 20 modulating functions are quite similar to those presented in Figure 8, which will be discussed later.

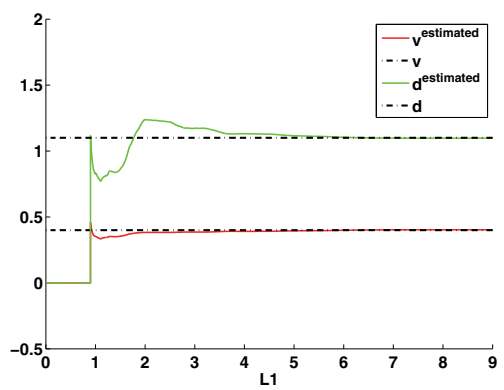

FIG. 8. Estimated $d$ and $\nu$ with $3 \%$ noise with different values of $L_{1}$ when $d=1.1, \nu=$ $0.4, \alpha=1.8$, and $\Delta x=\frac{1}{2100}$.

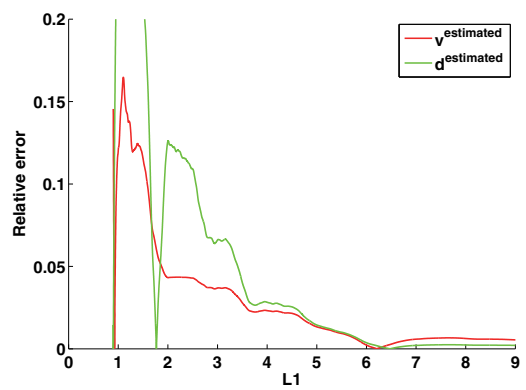

FIG. 9. Relative errors of $\nu$ and $d$ with $3 \%$ noise with different values of $L_{1}$ when $d=$ $1.1, \nu=0.4, \alpha=1.8$, and $\Delta x=\frac{1}{2100}$. 
TABLE 4

Relative errors of $\nu$ and $d$, where $\nu(x)=2(\sin (\pi x)+1), d(x)=5 \exp (-4 x), \alpha=1.8$, and $\Delta x=\frac{1}{2100}$ with $2 \%$ noise.

\begin{tabular}{ccc}
\hline \hline $\begin{array}{c}\text { Number of } \\
\text { modulating functions }\end{array}$ & $\begin{array}{c}\text { Relative errors } \\
\frac{\|-\tilde{\nu}\|_{2}}{\|\nu\|_{2}}\end{array}$ & $\frac{\|d-\tilde{d}\|_{2}}{\|d\|_{2}}$ \\
\hline 11 & 0.127 & 0.213 \\
12 & 0.159 & 0.236 \\
13 & 0.157 & 0.235 \\
14 & 0.150 & 0.230 \\
\hline
\end{tabular}

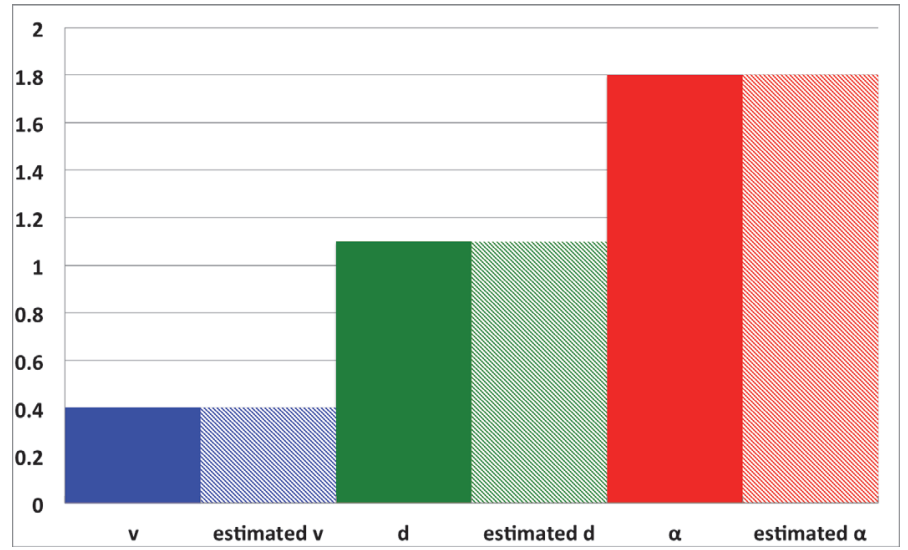

FIG. 10. A comparison between the exact and the estimated parameters with five modulating functions when adding a $3 \%$ noise to the measurements.

Estimating $\boldsymbol{\nu}, \boldsymbol{d}$, and $\boldsymbol{\alpha}$. Now, we use the combined Newton's and MFM to estimate all three parameters simultaneously. We set the exact values of the average velocity $\nu=0.4$, the dispersion coefficient $d=1.1$, the differentiation order $\alpha=1.8$, the length of the integration interval $L=9$, and the initial guess $\alpha_{o}=1.5$.

Noisy case. Figure 10 represents the estimated parameters using five modulating functions, where the noise level is $3 \%$.

In Figure 11, a comparison under different noise levels 1\%, 3\%, 5\%, 10\% between the exact values of the parameters and the estimated values is given. From this figure, it can be seen that the results are stable and remain reasonable even if adding $10 \%$ noise to the measurements. Figure 12 represents the results obtained with a different number of modulating functions when adding a $3 \%$ white Gaussian noise to the measurements. Even with a different number of modulating functions the errors are small and the results are quite satisfactory. This is confirmed in Table 5, where the relative errors are less than $2 \%$ for all parameters. However, it is noted that the number of the modulating functions has an effect on the stability and the accuracy of the presented algorithm. This problem will be discussed in the next section.

Estimating $\nu, d$, and $\alpha$ using interpolation. In practice it is difficult to measure the data over the whole domain. Therefore, in this part we use the measurements of the concentration and the flux at few locations, $x_{i}=\frac{L}{7} i$ :

$$
c\left(x_{i}, T\right)=g_{1}\left(x_{i}\right), \quad \frac{\partial c\left(x_{i}, T\right)}{\partial t}=g_{2}\left(x_{i}\right), \quad 0<x_{i}<L, \quad i=1,2, \ldots, 7,
$$




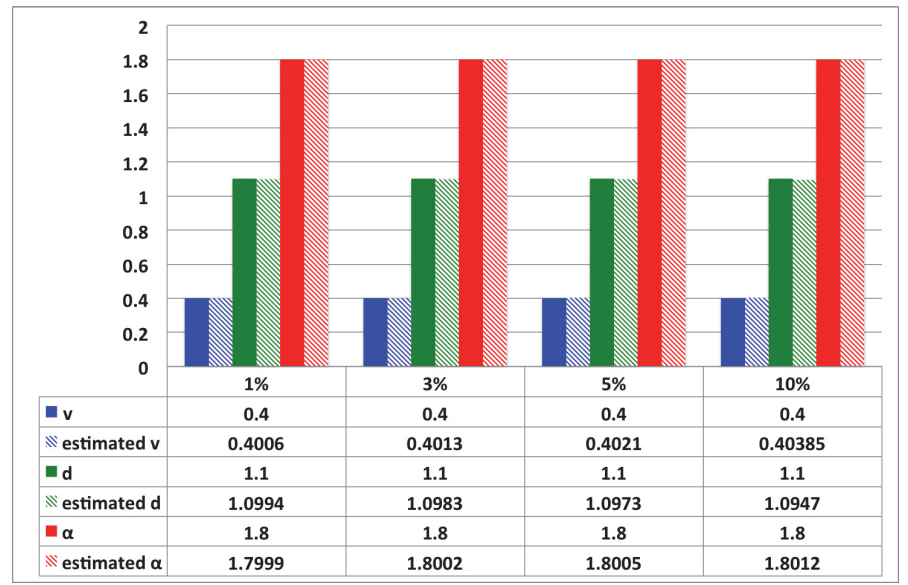

FIG. 11. Estimated parameters obtained with five modulating functions and different noise levels.

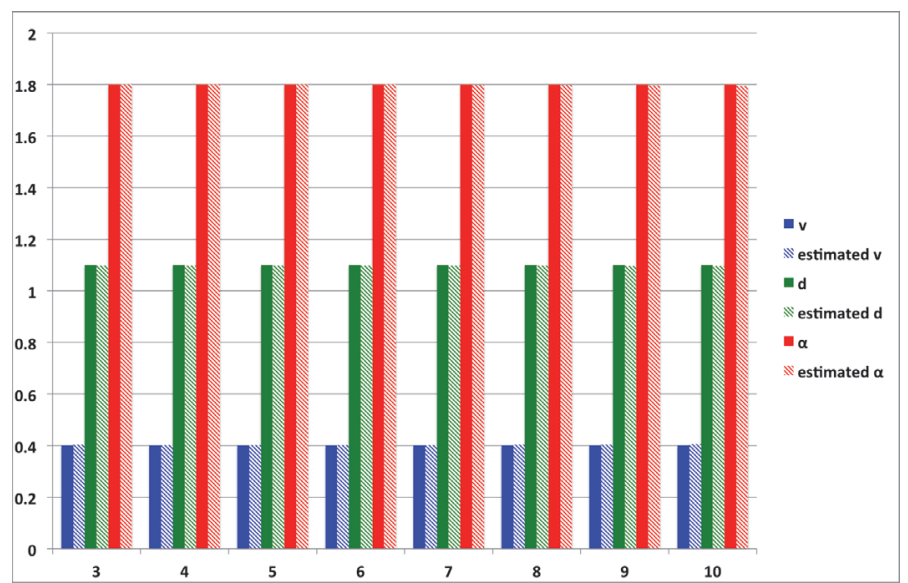

FIG. 12. Estimated parameters with a different number of modulating functions.

then use Legendre interpolation to approximate the measurements over the whole domain. We set the exact values of the average velocity $\nu=0.5$, the dispersion coefficient $d=1$, the differentiation order $\alpha=1.6$, the final time $T=1$, the length of the integration interval $L=40, b=3$, and the initial guess $\alpha_{o}=1.4$.

Noisy case. In Figure 13, the estimated $\nu$ and $d$ when adding a $3 \%$ white Gaussian noise to the measurements. Three modulating functions are used and the length of the integration interval $\left[0, L_{1}\right]$ has been increased. The corresponding relative errors are given in Figure 14. From these figures we note that the results are satisfactory and the relative errors are less than $6 \%$ when $L_{1}=40$ and less than $10 \%$ when $L_{1}$ is between 30 and 40. However, when we estimate all three unknowns $\nu, d$, and $\alpha$ the relative errors were slightly greater. Figure 15 represents the estimated parameters using five modulating functions, where the noise level is 3\%. In Figure 16, the estimated parameters with different noise levels and the corresponding relative errors are given in Figure 17. As we can see the relative errors for all three parameters are less than $4 \%$ when adding $1 \%$ noise and less than $6 \%$ for $3 \%$ noisy measurements. 
TABLE 5

$$
d=1.1, \alpha=1.8, \nu=0.4 \text {, and } \Delta x=\frac{1}{2100}, 3 \% \text { noise. }
$$

\begin{tabular}{ccc}
\hline \hline $\begin{array}{c}\text { Number of } \\
\text { modulating functions }\end{array}$ & $\begin{array}{c}\text { Estimated value } \\
\hat{a}=(\hat{\nu}, \hat{d}, \hat{\alpha})\end{array}$ & $\begin{array}{c}\text { Relative error } \\
\left(\frac{\|\nu-\hat{\nu}\|_{2}}{\|\nu\|_{2}}, \frac{\|d-\hat{d}\|_{2}}{\|d\|_{2}}, \frac{\|\alpha-\hat{\alpha}\|_{2}}{\|\alpha\|_{2}}\right)\end{array}$ \\
\hline 3 & $(0.403,1.098,1.799)$ & $(0.74 \mathrm{E}-2,0.19 \mathrm{E}-2,0.07 \mathrm{E}-2)$ \\
4 & $(0.406,1.098,1.799)$ & $(0.44 \mathrm{E}-2,0.15 \mathrm{E}-2,0.001 \mathrm{E}-2)$ \\
5 & $(0.401,1.098,1.800)$ & $(0.33 \mathrm{E}-2,0.15 \mathrm{E}-2,0.01 \mathrm{E}-2)$ \\
6 & $(0.402,1.098,1.800)$ & $(0.40 \mathrm{E}-2,0.18 \mathrm{E}-2,0.03 \mathrm{E}-2)$ \\
7 & $(0.402,1.098,1.799)$ & $(0.57 \mathrm{E}-2,0.22 \mathrm{E}-2,0.09 \mathrm{E}-2)$ \\
8 & $(0.403,1.097,1.797)$ & $(0.83 \mathrm{E}-2,0.27 \mathrm{E}-2,0.17 \mathrm{E}-2)$ \\
9 & $(0.405,1.097,1.795)$ & $(1.13 \mathrm{E}-2,0.31 \mathrm{E}-2,0.26 \mathrm{E}-2)$ \\
10 & $(0.406,1.096,1.794)$ & $(1.43 \mathrm{E}-2,0.33 \mathrm{E}-2,0.24 \mathrm{E}-2)$ \\
\hline
\end{tabular}

Moreover, in Tables 6 and 7 are the estimated parameters with $1 \%$ and $2 \%$ noise with a different number of modulating functions. It is noted that when using four and five modulating functions the results are more accurate than when increasing the number of modulating functions. As mentioned before the number of modulating functions has an effect on the accuracy of the algorithm and further investigation is needed.

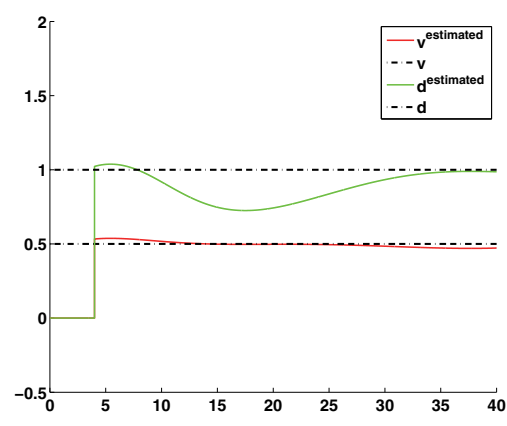

FIG. 13. Exact and estimated $\nu$ with $3 \%$ noise when $L_{1}=40$ and $\Delta x=\frac{1}{2000}$.

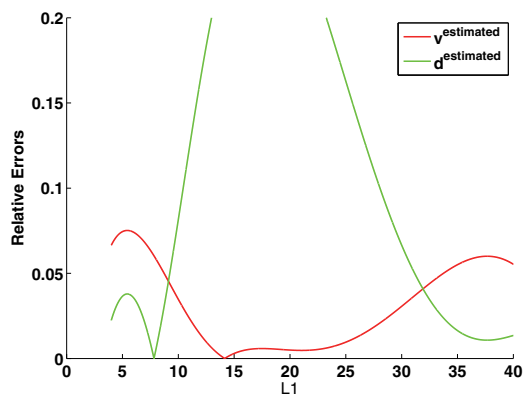

FIG. 14. The exact and the estimated $d$ with a different number of modulating functions with $3 \%$ noise when $L_{1}=40$ and $\Delta x=$ $\frac{1}{2000}$.

Remark 6.1. It is important to point out that in the noisy case the accuracy and stability of the system given in (3.9) does not depend only on the noise, but also depends on the sensitivity of the system, which is the condition number of the matrix. Since the condition number depends on both the input and the output of our system, the choice of the modulating functions is critical and should be chosen to give a small condition number.

Remark 6.2. We would like to point out that when estimating variable coefficients we may have some ill conditioning problems as we need to increase the number of basis functions. Moreover, we need to evaluate the fractional derivative of the basis functions, Therefore, in our numerical examples we have used a polynomial basis just to simplify the calculations and to avoid computing the fractional derivatives numerically; this is one of the important advantages of using these basis functions. However, the case where both coefficients are nonpolynomial may require the use of another type of basis, but this will require the numerical computation of the fractional derivatives of the basis functions. This will be investigated in future work. 


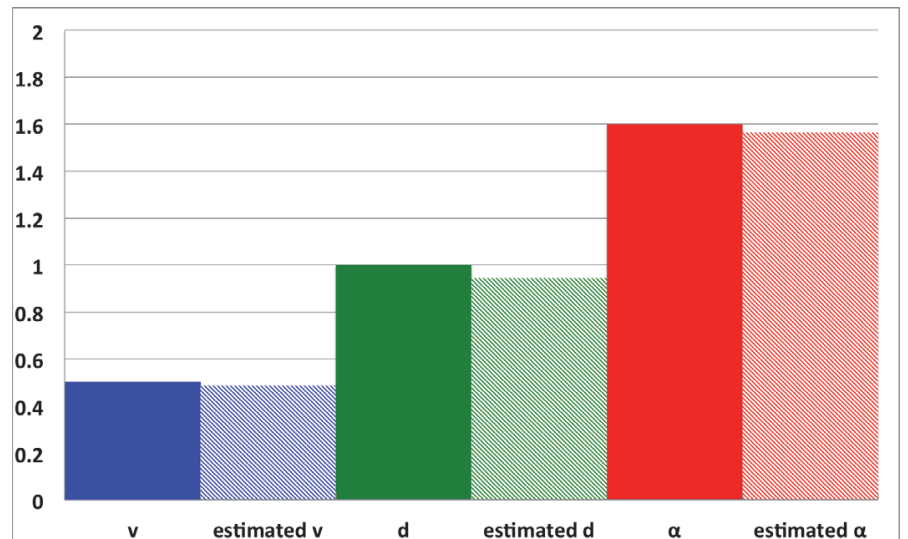

FIG. 15. A comparison between the exact and the estimated parameters with five modulating functions when adding a $3 \%$ noise to the measurements.

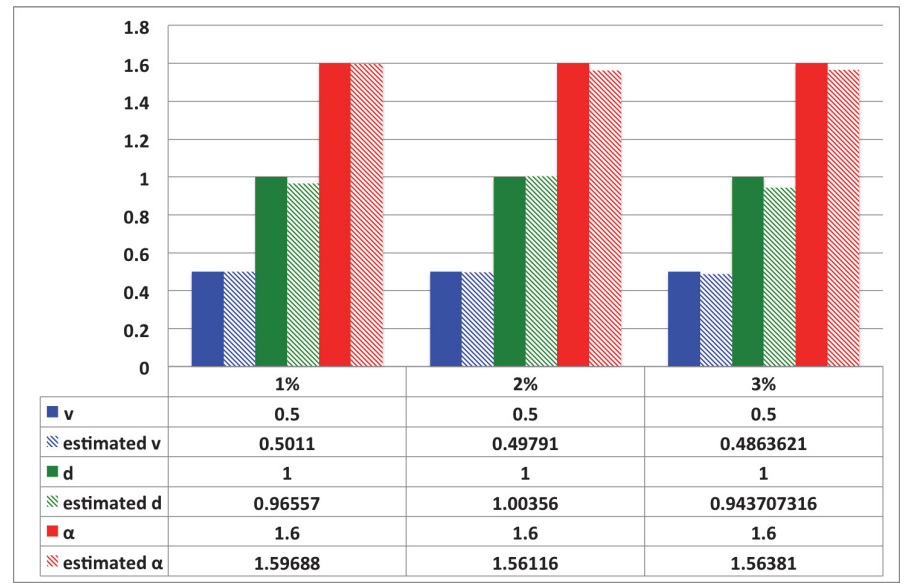

FIG. 16. Estimated parameters obtained with five modulating functions and different noise levels.

Example 6.2. We consider (1.1) with initial and Dirichlet boundary conditions given in (1.2), where $g_{0}(x)=10 x^{2}-x^{3}$ and

$$
r(x, t)= \begin{cases}t^{2}\left[\nu(x)\left(20 x-3 x^{2}\right)-d(x)\left(\frac{10 \Gamma(3)}{\Gamma(3-\alpha)} x^{1-\alpha}-\frac{\Gamma(4)}{\Gamma(4-\alpha)} x^{2-\alpha}\right)\right] & \\ 0, & 0<x<10, \\ 02 t\left(10 x^{2}-x^{3}\right), & x=0,10 .\end{cases}
$$

The exact solution of the forward problem is $c(x, t)=t^{2}\left(10 x^{2}-x^{3}\right)$ and the flux is $\frac{\partial c(x, t)}{\partial t}=2 t\left(10 x^{2}-x^{3}\right)$.

In this example, we set the average velocity $\nu=0.3$, the dispersion coefficient $d=1.1$, the differentiation order $\alpha=1.3$, and the final time $T=2$. In Figure 18, we estimated $\nu$ and $d$ using three modulating functions when adding a $2 \%$ noise to the measurements, and the corresponding relative errors are given in Figure 19. Figure 20 represents the estimated $\nu, d$, and $\alpha$ when using four modulating functions with different noise levels. On the other hand, Figure 21 represents the estimated parameters with a different number of modulating functions. As we can see in both cases the 


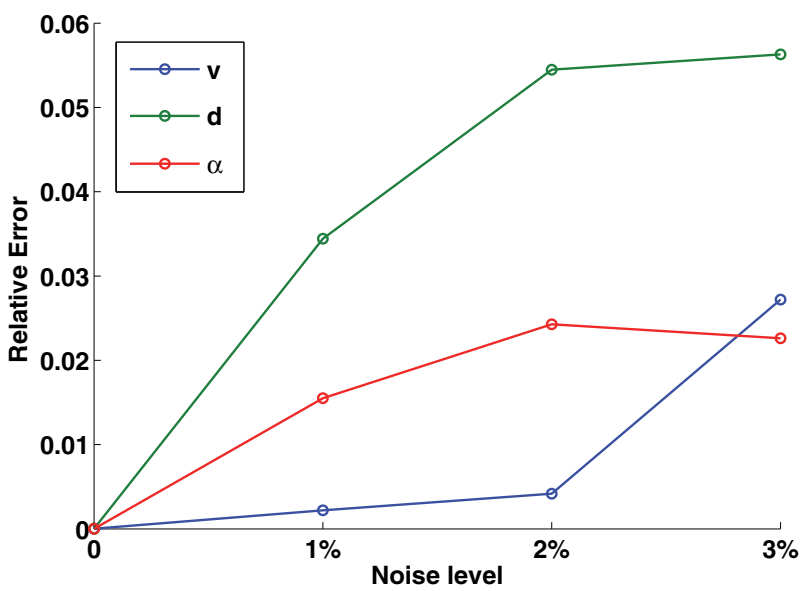

FIG. 17. Relative errors for the estimated parameters with five modulating functions with different noise levels.

TABLE 6

$d=1, \alpha=1.6, \nu=0.5$, and $\Delta x=\frac{1}{2000}, 1 \%$ noise.

\begin{tabular}{ccc}
\hline \hline $\begin{array}{c}\text { Number of } \\
\text { modulating functions }\end{array}$ & $\begin{array}{c}\text { Estimated value } \\
\hat{a}=(\hat{\nu}, \hat{d}, \hat{\alpha})\end{array}$ & $\begin{array}{c}\text { Relative error } \\
\left(\frac{\|\nu-\hat{\nu}\|_{2}}{\|\nu\|_{2}}, \frac{\|d-\hat{d}\|_{2}}{\|d\|_{2}}, \frac{\|\alpha-\hat{\alpha}\|_{2}}{\|\alpha\|_{2}}\right)\end{array}$ \\
\hline 3 & $(0.478,1.020,1.620)$ & $(4.40 \mathrm{E}-2,2.04 \mathrm{E}-2,1.26 \mathrm{E}-2)$ \\
4 & $(0.489,0.990,1.597)$ & $(2.12 \mathrm{E}-2,0.97 \mathrm{E}-2,0.19 \mathrm{E}-2)$ \\
5 & $(0.501,0.966,1.575)$ & $(0.22 \mathrm{E}-2,3.44 \mathrm{E}-2,1.55 \mathrm{E}-2)$ \\
6 & $(0.513,0.946,1.555)$ & $(2.61 \mathrm{E}-2,5.43 \mathrm{E}-2,2.81 \mathrm{E}-2)$ \\
7 & $(0.525,0.930,1.536)$ & $(5.05 \mathrm{E}-2,6.97 \mathrm{E}-2,3.98 \mathrm{E}-2)$ \\
\hline
\end{tabular}

TABLE 7

$d=1.1, \alpha=1.8, \nu=0.4$, and $\Delta x=\frac{1}{2000}, 2 \%$ noise.

\begin{tabular}{ccc}
\hline \hline $\begin{array}{c}\text { Number of } \\
\text { modulating functions }\end{array}$ & $\begin{array}{c}\text { Estimated value } \\
\hat{a}=(\hat{\nu}, \hat{d}, \hat{\alpha})\end{array}$ & $\begin{array}{c}\text { Relative error } \\
\left(\frac{\|\nu-\hat{\nu}\|_{2}}{\|\nu\|_{2}}, \frac{\|d-\hat{d}\|_{2}}{\|d\|_{2}}, \frac{\|\alpha-\hat{\alpha}\|_{2}}{\|\alpha\|_{2}}\right)\end{array}$ \\
\hline 3 & $(0.446,1.079,1.664)$ & $(10.9 \mathrm{E}-2,7.94 \mathrm{E}-2,3.99 \mathrm{E}-2)$ \\
4 & $(0.471,1.004,1.611)$ & $(5.89 \mathrm{E}-2,0.36 \mathrm{E}-2,0.07 \mathrm{E}-2)$ \\
5 & $(0.498,0.946,1.561)$ & $(0.417 \mathrm{E}-2,5.45 \mathrm{E}-2,2.42 \mathrm{E}-2)$ \\
6 & $(0.528,0.904,1.514)$ & $(5.51 \mathrm{E}-2,9.58 \mathrm{E}-2,5.38 \mathrm{E}-2)$ \\
7 & $(0.559,0.878,1.470)$ & $(11.9 \mathrm{E}-2,12.2 \mathrm{E}-2,8.10 \mathrm{E}-2)$ \\
\hline
\end{tabular}

results are satisfactory which is conformed by the errors given in Table 8 . In Table 9, we estimated the parameters with a different initial guess $\alpha_{0}=1.2,1.35,1.37,1.4,1.5$, and 1.6. As expected, the algorithm converges to the exact solution when the initial guess is around the true value of $\alpha$. However, when we choose $\alpha_{0}=1.5$ or 1.6 , which is more then $15 \%$ far from the exact value, the algorithm does not converge to the exact solution.

7. Discussion. A two stage algorithm has been used to estimate the coefficients and the differentiation order for a fractional differential equation. In the proposed approach, we take advantage of the properties of the modulating functions to overcome the difficulties in estimating the differentiation order. The main advantage is that we 


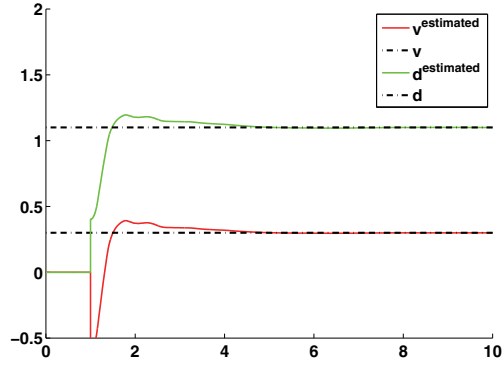

Fig. 18. Estimated $d$ and $\nu$ with $2 \%$ noise with different values of $L_{1}$ when $d=$ $1.1, \nu=0.3, \alpha=1.3$, and $\Delta x=\frac{1}{3000}$.

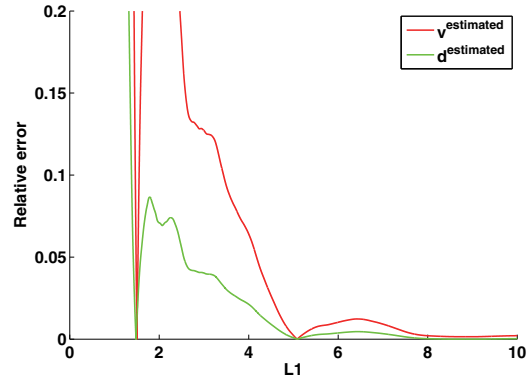

FIG. 19. Relative errors of $\nu$ and $d$ with $2 \%$ noise with different values of $L_{1}$ when $d=$ $1.1, \nu=0.3, \alpha=1.3$, and $\Delta x=\frac{1}{3000}$.

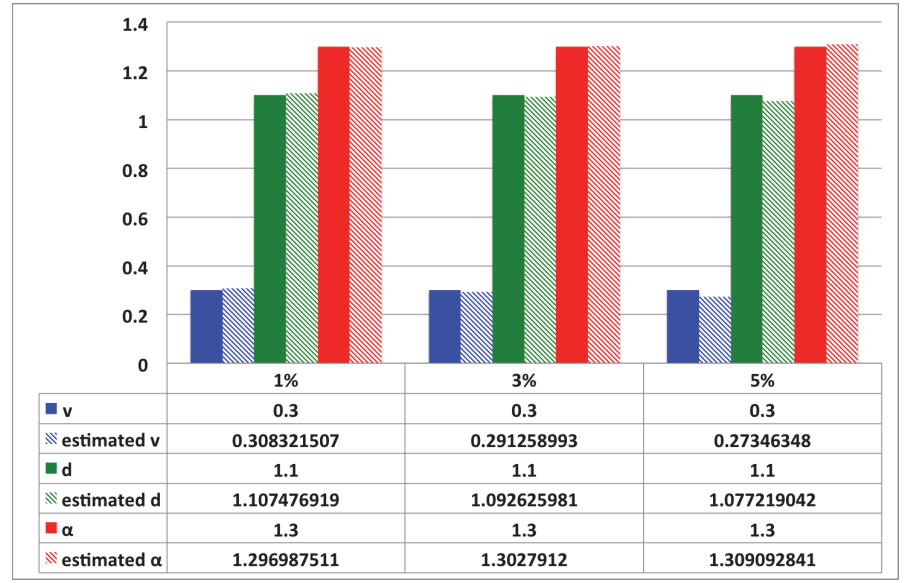

FIG. 20. Estimated parameters obtained with four modulating functions and different noise levels.

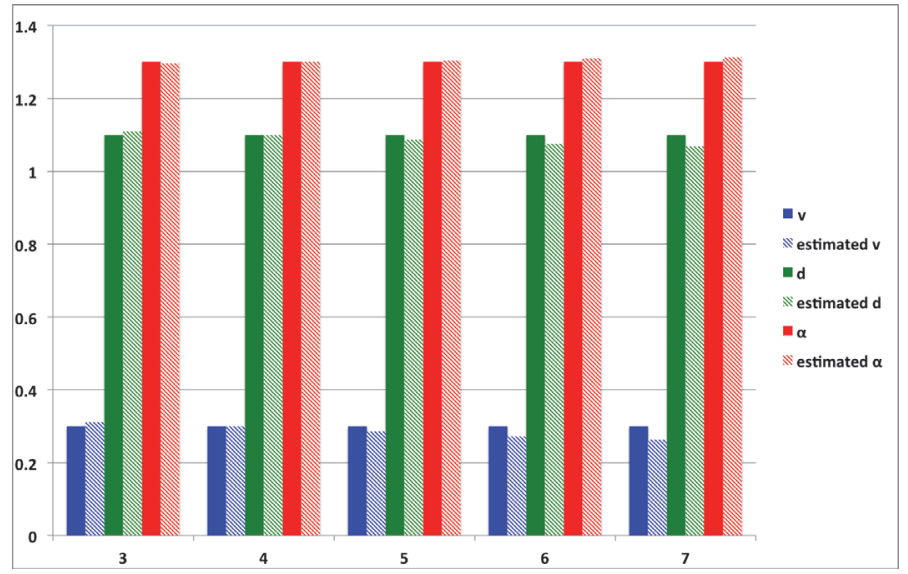

FIG. 21. Estimated parameters with a different number of modulating functions.

Copyright (C) by SIAM. Unauthorized reproduction of this article is prohibited. 
TABLE 8

$d=1.1, \alpha=1.3, \nu=0.3$, and $\Delta x=\frac{1}{3000}, 2 \%$ noise.

\begin{tabular}{ccc}
\hline \hline $\begin{array}{c}\text { Number of } \\
\text { modulating functions }\end{array}$ & $\begin{array}{c}\text { Estimated value } \\
\hat{a}=(\hat{\nu}, \hat{d}, \hat{\alpha})\end{array}$ & $\begin{array}{c}\text { Relative error } \\
\left(\frac{\|\nu-\hat{\nu}\|_{2}}{\|\nu\|_{2}}, \frac{\|d-\hat{d}\|_{2}}{\|d\|_{2}}, \frac{\|\alpha-\hat{\alpha}\|_{2}}{\|\alpha\|_{2}}\right)\end{array}$ \\
\hline 3 & $(0.311,1.110,1.296)$ & $(3.64 \mathrm{E}-2,0.899 \mathrm{E}-2,0.308 \mathrm{E}-2)$ \\
4 & $(0.300,1.100,1.299)$ & $(0.07 \mathrm{E}-2,0.003 \mathrm{E}-2,0.01 \mathrm{E}-2)$ \\
5 & $(0.285,1.087,1.305)$ & $(5.01 \mathrm{E}-2,1.18 \mathrm{E}-2,0.4 \mathrm{E}-2)$ \\
6 & $(0.272,1.076,1.310)$ & $(9.34 \mathrm{E}-2,2.22 \mathrm{E}-2,0.77 \mathrm{E}-2)$ \\
7 & $(0.264,1.068,1.313)$ & $(12.1 \mathrm{E}-2,2.89 \mathrm{E}-2,10.2 \mathrm{E}-2)$ \\
\multicolumn{4}{c}{ TABLE 9} \\
\\
\multicolumn{4}{c}{$\alpha=1.1, \alpha=1.3, \nu=0.3$, and $\Delta x=\frac{1}{3000}, 2 \%$ noise. } \\
\hline \hline Initial guess & Estimated value \\
$\alpha_{0}$ & $\hat{a}=(\hat{\nu}, \hat{d}, \hat{\alpha})$ & $\left(\frac{\|\nu-\hat{\nu}\|_{2}}{\|\nu\|_{2}}, \frac{\|d-\hat{d}\|_{2}}{\|d\|_{2}}, \frac{\|\alpha-\hat{\alpha}\|_{2}}{\|\alpha\|_{2}}\right)$ \\
\hline 1.2 & $(0.299,1.1,1.3)$ & $(0.002 \mathrm{E}-2,0.03 \mathrm{E}-2,0.002 \mathrm{E}-2)$ \\
1.35 & $(0.265,1.07,1.313)$ & $(11.6 \mathrm{E}-2,2.75 \mathrm{E}-2,0.97 \mathrm{E}-2)$ \\
1.37 & $(0.265,1.07,1.297)$ & $(11.6 \mathrm{E}-2,2.75 \mathrm{E}-2,0.97 \mathrm{E}-2)$ \\
1.4 & $(0.266,1.07,1.312)$ & $(11.4 \mathrm{E}-2,2.72 \mathrm{E}-2,0.95 \mathrm{E}-2)$ \\
1.5 & $(0.163,0.766,1.62)$ & $(45.7 \mathrm{E}-2,30.3 \mathrm{E}-2,24.6 \mathrm{E}-2)$ \\
1.6 & $(0.163,0.766,1.62)$ & $(45.7 \mathrm{E}-2,30.3 \mathrm{E}-2,24.6 \mathrm{E}-2)$ \\
\hline
\end{tabular}

simplify the Newton's algorithm by reducing the number of variables in the nonlinear equation problem and the efficient computation of the gradient. The efficiency and the robustness against corrupting noise with a different number of modulating functions have been confirmed by numerical examples. It is noted that the choice and the number of modulating functions can affect the accuracy of the proposed algorithm. As we can see in Figures 22 and 23, for 3 to up to 20 modulating functions the relative errors vary, but are still less than $10 \%$. It is noted that the numerical accuracy can become worse if the number of modulating functions increases. This is because the stability of the algebraic system given in (3.10) depends on the modulating functions. Indeed, as we increase the number of modulating functions the number of equations increases. In all cases, the results are stable and remain reasonable even for up to 20 modulating functions and the presented algorithm works well with a reasonable number of modulating functions, which is further confirmed by the errors in Table 5 .

Finally, we briefly comment on the length of the integration interval. In Tables 10 and 11, we present the relative errors when estimating the average velocity and the dispersion coefficient with different integration intervals. We observe that the length of the integration interval also has an effect on the accuracy of the performed algorithm. However, the relative errors are still reasonable and less than $1 \%$ for $L_{1}=5$. In fact, there is an optimal value for the length of the integration interval and further investigation is needed. Further, in Table 12, we present the relative errors when estimating all three parameters.

8. Conclusion. In this paper, we have presented a new approach to estimate the coefficients and the differentiation order in a space-fractional advection-dispersion equation. First, we have estimated the dispersion coefficient and the average velocity by applying the MFM which transformed the coefficients identification problem into solving a linear system of algebraic equations. Then, these estimations of the unknown coefficients are robust against high frequency noises. Second, the MFM has been combined with Newton's iterative algorithm to estimate the average velocity, the 


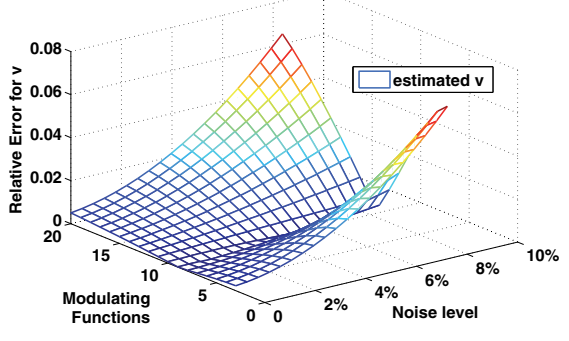

FIG. 22. The relative errors of $\nu$.

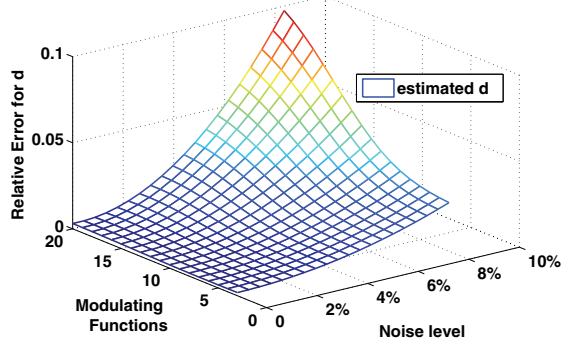

FIG. 23. The relative errors of $d$.

TABLE 10

Estimating $d$ when the exact values are $d=1.1, \alpha=1.8, \nu=0.4$, and $\Delta x=\frac{1}{2100}, 3 \%$ noise on both measurements.

\begin{tabular}{cccccc}
\hline \hline Number of & \multicolumn{5}{c}{ Relative errors } \\
modulating functions & $L_{1}=5$ & $L_{1}=6$ & $L_{1}=7$ & $L_{1}=8$ & $L_{1}=9$ \\
\hline 3 & $0.81 \mathrm{E}-2$ & $0.40 \mathrm{E}-2$ & $0.20 \mathrm{E}-2$ & $0.20 \mathrm{E}-2$ & $0.17 \mathrm{E}-2$ \\
4 & $0.76 \mathrm{E}-2$ & $0.40 \mathrm{E}-2$ & $0.18 \mathrm{E}-2$ & $0.18 \mathrm{E}-2$ & $0.16 \mathrm{E}-2$ \\
5 & $0.67 \mathrm{E}-2$ & $0.38 \mathrm{E}-2$ & $0.14 \mathrm{E}-2$ & $0.14 \mathrm{E}-2$ & $0.14 \mathrm{E}-2$ \\
6 & $0.56 \mathrm{E}-2$ & $0.35 \mathrm{E}-2$ & $0.08 \mathrm{E}-2$ & $0.08 \mathrm{E}-2$ & $0.11 \mathrm{E}-2$ \\
7 & $0.43 \mathrm{E}-2$ & $0.31 \mathrm{E}-2$ & $0.03 \mathrm{E}-2$ & $0.03 \mathrm{E}-2$ & $0.08 \mathrm{E}-2$ \\
\hline
\end{tabular}

TABLE 11

Estimating $\nu$ when the exact values are $d=1, \alpha=1.8, \nu=0.5$, and $\Delta x=\frac{1}{2100}, 2 \%$ noise on both measurements.

\begin{tabular}{cccccc}
\hline \hline Number of & \multicolumn{5}{c}{ Relative errors } \\
modulating functions & $L_{1}=5$ & $L_{1}=6$ & $L_{1}=7$ & $L_{1}=8$ & $L_{1}=9$ \\
\hline 3 & $1.13 \mathrm{E}-2$ & $0.60 \mathrm{E}-2$ & $0.05 \mathrm{E}-2$ & $0.05 \mathrm{E}-2$ & $0.07 \mathrm{E}-2$ \\
4 & $1.07 \mathrm{E}-2$ & $0.56 \mathrm{E}-2$ & $0.02 \mathrm{E}-2$ & $0.02 \mathrm{E}-2$ & $0.08 \mathrm{E}-2$ \\
5 & $0.97 \mathrm{E}-2$ & $0.49 \mathrm{E}-2$ & $0.02 \mathrm{E}-2$ & $0.02 \mathrm{E}-2$ & $0.08 \mathrm{E}-2$ \\
6 & $0.83 \mathrm{E}-2$ & $0.39 \mathrm{E}-2$ & $0.07 \mathrm{E}-2$ & $0.07 \mathrm{E}-2$ & $0.08 \mathrm{E}-2$ \\
7 & $0.67 \mathrm{E}-2$ & $0.28 \mathrm{E}-2$ & $0.12 \mathrm{E}-2$ & $0.12 \mathrm{E}-2$ & $0.07 \mathrm{E}-2$ \\
\hline
\end{tabular}

TABLE 12

When estimating all parameters $d=1.1, \alpha=1.8, \nu=0.4$, and $\Delta x=\frac{1}{2100}, 2 \%$ noise on both measurements.

\begin{tabular}{|c|c|c|c|c|}
\hline $\bar{M}$ & $L_{1}=7.5$ & $\begin{array}{c}\text { Relative error } \\
L_{1}=8\end{array}$ & $L_{1}=8.5$ & $L_{1}=9$ \\
\hline 3 & $(26.83 \mathrm{E}-2,4.14 \mathrm{E}-2,4.91 \mathrm{E}-2)$ & $(13.48 \mathrm{E}-2,2.53 \mathrm{E}-2,2.28 \mathrm{E}-2)$ & & \\
\hline 4 & $(23.63 \mathrm{E}-2,3.86 \mathrm{E}-2,4.32 \mathrm{E}-2)$ & $(10.07 \mathrm{E}-2,2.31 \mathrm{E}-2,1.60 \mathrm{E}-2)$ & $(4.83 \mathrm{E}-2,1.31 \mathrm{E}-2,0.47 \mathrm{E}-2)$ & $9 \mathrm{E}-2,0.89 \mathrm{E}-2,0.14 \mathrm{E}-2)$ \\
\hline 5 & $(20.40 \mathrm{E}-2,3.58 \mathrm{E}-2,3.77 \mathrm{E}-2)$ & $(7.23 \mathrm{E}-2,1.85 \mathrm{E}-2,0.78 \mathrm{E}-2)$ & $(3.62 \mathrm{E}-2,1.25 \mathrm{E}-2,0.29 \mathrm{E}-2)$ & $(1.76 \mathrm{E}-2,0.93 \mathrm{E}-2,0.18 \mathrm{E}-2)$ \\
\hline 6 & $(17.02 \mathrm{E}-2,3.32 \mathrm{E}-2,3.24 \mathrm{E}-$ & $(4.79 \mathrm{E}-2,1.69 \mathrm{E}-2,0.77 \mathrm{E}-2)$ & $(3.44 \mathrm{E}-2,1.38 \mathrm{E}-2,0.41 \mathrm{E}-2)$ & $(2.19 \mathrm{E}-2,1.15 \mathrm{E}-2,0.06 \mathrm{E}-2)$ \\
\hline 7 & $(13.50 \mathrm{E}-2,3.11 \mathrm{E}-2,2.73 \mathrm{E}-2)$ & $(0.27 \mathrm{E}-2,1.68 \mathrm{E}-2,0.55 \mathrm{E}-2)$ & $(4.23 \mathrm{E}-2,1.64 \mathrm{E}-2,0.76 \mathrm{E}-2)$ & $(3.36 \mathrm{E}-2,1.44 \mathrm{E}-2,0.48 \mathrm{E}-2)$ \\
\hline
\end{tabular}

dispersion coefficient, and the differentiation order simultaneously, where the first order derivatives with respect to $\alpha$ of the dispersion coefficient and the average velocity have also been estimated using a modulating function approach, which simplifies the calculation of the gradient. Moreover, the local convergence of the presented method has been proved. Finally, numerical simulations have been performed and the results have shown the effectiveness of the proposed algorithm. In these numerical examples, we have chosen polynomial modulating functions, for which the fractional derivative is known analytically. For other types of modulating functions further studies will be 
conducted.

Acknowledgment. The authors would like to thank the anonymous reviewers for their valuable comments, which helped significantly improve the quality of this manuscript.

\section{REFERENCES}

[1] A. N. Bondarenko and D. S. Ivaschenko, Generalized sommerfeld problem for time fractional diffusion equation: Analytical and numerical approach, J. Inverse Ill-Posed Probl., 17 (2009), pp. 321-335.

[2] A. N. Bondarenko And D. S. Ivaschenko, Numerical methods for solving inverse problems for time fractional diffusion equation with variable coefficient, J. Inverse Ill-Posed Probl., 17 (2009), pp. 419-440.

[3] H. Brunner, L. Ling, and M. Yamamoto, Numerical simulations of $2 d$ fractional subdiffusion problems, J. Comput. Phys., 229 (2010), pp. 6613-6632.

[4] G. ChI, G. LI, AND X. JIA, Numerical inversions of a source term in the FADE with a dirichlet boundary condition using final observations, Comput. Math. Appl., 62 (2011), pp. 16191626 .

[5] T. B. Co AND S. Ungarala, Batch scheme recursive parameter estimation of continuous-time system using the modulating functions method, Automatica J. IFAC, 33 (1997), pp. 11851191.

[6] G. Fedele and L. Coluccio, A recursive scheme for frequency estimation using the modulating functions method, Appl. Math. Comput., 216 (2010), pp. 1393-1400.

[7] S. Fomin, V. Chugunov, And T. Hashida, Application of fractional differential equations for modeling the anomalous diffusion of contaminant from fracture into porous rock matrix with bordering alteration zone, Transp. Porous Media, 81 (2010), pp. 187-205.

[8] Z.-J. Fu, W. Chen, AND H.-T. YANG, Boundary particle method for laplace transformed time fractional diffusion equations, J. Comput. Phys., 235 (2013), pp. 52-66.

[9] T. JANICZEK, Generalization of modulating functions method into the fractional differential equations, Bull. Polish Acad. Sci. Tech. Sci., 58 (2010), pp. 593-599.

[10] B. Jin And W. Rundell, An inverse problem for a one-dimensional time-fractional diffusion problem, Inverse Problems, 28 (2012), 075010.

[11] C. T. Kelley, Iterative Methods for Linear and Nonlinear Equations, Frontiers Appl. Math. 16, SIAM, Philadelphia, 1995.

[12] D. Y. Liu, O. Gibaru, and W. Perruquetti, Parameters estimation of a noisy sinusoidal signal with time-varying amplitude, in 19th Mediterranean Conference on Control and Automation (MED'11), Corfu, Greece, 2011, pp. 570-575.

[13] D.-Y. LiU And T.-M. LALEG-KiRATi, Robust fractional order differentiators using generalized modulating functions method, Signal Process., 107 (2015), pp. 395-406.

[14] D.-Y. Liu, T.-M. Laleg-Kirati, O. Gibaru, and W. Perruquetti, Identification of fractional order systems using modulating functions method, in the 2013 American Control Conference, Washington, DC, 2013, pp. 1679-1684.

[15] D.-Y. Liu, T.-M. Laleg-Kirati, W. Perruquetti, and O. Gibaru, Non-asymptotic state estimation for a class of linear time-varying systems with unknown inputs, in 19th World Congress of the International Federation of Automatic Control, Cape Town, South Africa, 2014, pp. 3732-3738.

[16] F. MAinaRdi, The fundamental solutions for the fractional diffusion-wave equation, Appl. Math. Lett., 9 (1996), pp. 23-28.

[17] L. Miller and M. Yamamoto, Coefficient inverse problem for a fractional diffusion equation, Inverse Problems, 29 (2013), 075013.

[18] A. Pedas and E. Tamme, Numerical solution of nonlinear fractional differential equations by spline collocation methods, J. Comput. Appl. Math., 255 (2014), pp. 216-230.

[19] I. Podlubny, Fractional Differential Equations, Academic Press, San Diego, 1999.

[20] H. A. Preising And D. W. T. Rippin, Theory and application of the modulating function method $i$. Review and theory of the method and theory of the spline-type modulating functions, Comput. Chem. Eng., 17 (1993), pp. 1-16.

[21] W. Rundell, X. Xu, AND L. Zuo, The determination of an unknown boundary condition in a fractional diffusion equation, Appl. Anal., 92 (2013), pp. 1511-1526.

[22] M. S. Sadabadi, M. Shafiee, and M. Karrari, System identification of two-dimensional continuous-time systems using wavelets as modulating functions, ISA Trans., 47 (2008), pp. 256-266.

Copyright $@$ by SIAM. Unauthorized reproduction of this article is prohibited. 
[23] R. Schumer, D. Benson, M. M. Meerschaert, and S. W. Wheatcraft, Eulerian derivation of the fractional advection-dispersion equation, J. Contam. Hydrol., 48 (2001), pp. 69-88.

[24] R. Schumer, M. Meerschaert, and B. Baeumer, Fractional advection-dispersion equations for modeling transport at the earth surface, J. Geophys. Res. Earth, 114 (2009), F00A07.

[25] M. Shinbrot, On the Analysis of Linear and Nonlinear Dynamic Systems from Transientresponse Data, National Advisory Committee for Aeronautics (NACA), Technical report, Technical Note 3288, Washington, DC, 1954.

[26] H. G. Sun, Y. Zhang, W. Chen, and D. M. Reeves, Use of a variable-index fractionalderivative model to capture transient dispersion in heterogeneous media, J. Contam. Hydrol., 157 (2014), pp. 47-58.

[27] S. Ungarala AND T. B. Co, Time-varying system identification using modulating functions and spline models with application to bio-processes, Comput. Chem. Eng., 24 (2000), pp. $2739-2753$.

[28] H. Wei, W. Chen, H. Sun, And X. Li, A coupled method for inverse source problem of spatial fractional anomalous diffusion equations, Inverse Probl. Sci. Eng., 18 (2010), pp. 945-956.

[29] X. Xiong, Q. Zhou, And Y. C. Hon, An inverse problem for fractional diffusion equation in 2-dimensional case: Stability analysis and regularization, J. Math. Anal. Appl., 393 (2012), pp. 185-199.

[30] D. Zhang, G. Li, G. Chi, X. JiA, And H. LI, Numerical identification of multi-parameters in the space fractional advection dispersion equation by final observations, J. Appl. Math., 2012 (2012), 740385.

Copyright (c) by SIAM. Unauthorized reproduction of this article is prohibited. 